\title{
The longest telomeres: a general signature of adult stem
cell compartments
}

\author{
Ignacio Flores, ${ }^{1}$ Andres Canela, ${ }^{1}$ Elsa Vera, ${ }^{1}$ Agueda Tejera, ${ }^{1}$ George Cotsarelis, ${ }^{2}$ \\ and María A. Blasco ${ }^{1,3}$ \\ ${ }^{1}$ Telomeres and Telomerase Group, Molecular Oncology Program, Spanish National Cancer Centre (CNIO), \\ Madrid E-28029, Spain; ${ }^{2}$ University of Pennsylvania School of Medicine, M8 Stellar-Chance Laboratories, \\ Philadelphia, Pennsylvania 19104, USA
}

\begin{abstract}
Identification of adult stem cells and their location (niches) is of great relevance for regenerative medicine. However, stem cell niches are still poorly defined in most adult tissues. Here, we show that the longest telomeres are a general feature of adult stem cell compartments. Using confocal telomere quantitative fluorescence in situ hybridization (telomapping), we find gradients of telomere length within tissues, with the longest telomeres mapping to the known stem cell compartments. In mouse hair follicles, we show that cells with the longest telomeres map to the known stem cell compartments, colocalize with stem cell markers, and behave as stem cells upon treatment with mitogenic stimuli. Using K15-EGFP reporter mice, which mark hair follicle stem cells, we show that GFP-positive cells have the longest telomeres. The stem cell compartments in small intestine, testis, cornea, and brain of the mouse are also enriched in cells with the longest telomeres. This constitutes the description of a novel general property of adult stem cell compartments. Finally, we make the novel finding that telomeres shorten with age in different mouse stem cell compartments, which parallels a decline in stem cell functionality, suggesting that telomere loss may contribute to stem cell dysfunction with age.
\end{abstract}

[Keywords: Telomeres; stem cell niches; telomerase; mouse; aging]

Supplemental material is available at http://www.genesdev.org.

Received July 27, 2007; revised version accepted December 19, 2007.

Telomeres are nucleoprotein structures that protect the ends of chromosomes from DNA repair and degradation (Chan and Blackburn 2002). In vertebrates, telomeres consist of TTAGGG repeats that are bound by a multiprotein complex known as "shelterin" or "the telosome," which has fundamental roles in the regulation of telomere length and protection (de Lange 2005). Proper telomere functioning requires both a minimum length of TTAGGG repeats and the integrity of the shelterin complex (de Lange 2005). Telomere length is maintained by telomerase, a reverse transcriptase that adds telomeric repeats de novo after each cell division, counteracting the end-replication problem in those cell types where it is expressed (Chan and Blackburn 2002). Telomerase is expressed in germ cells and in the stem cell compartment of several adult tissues, as well as in other adult non-stem cell populations that activate telomerase upon treatment with proliferative stimuli (i.e., lymphocytes, keratinocytes) (Ramirez et al. 1997; Harrington 2004;

${ }^{3}$ Corresponding author.

E-MAIL mblasco@cnio.es; FAX 34-91-732-8028.

Article published online ahead of print. Article and publication date are online at http://www.genesdev.org/cgi/doi/10.1101/gad.451008.
Lansdorp 2005; Flores et al. 2006). However, telomerase activity levels in human adult tissues are not sufficient to prevent progressive telomere shortening with age (Collins and Mitchell 2002). The main cause of telomere shortening is thought to be the incomplete replication of the telomeric DNA, which occurs with each cell division cycle, although other factors such as the action of exonucleases or processes associated with DNA metabolism could contribute to telomere degradation (Collins and Mitchell 2002; Blasco 2005). The fact that telomerase activity is rate-limiting for most tissues becomes apparent when considering the premature aging phenotype of late-generation telomerase-deficient mice (Blasco 2005), or of humans with decreased levels of telomerase, as in some cases of dyskeratosis congenita or aplastic anemia (Collins and Mitchell 2002; Mason et al. 2005). Of interest, adult stem cells divide very infrequently (Fuchs et al. 2004) and reside in protected microenvironments or niches (Moore and Lemischka 2006), suggesting a low rate of telomere erosion (loss of TTAGGG sequences from chromosome ends) throughout their lifetime.

The identification of adult stem cell compartments is essential for studying adult stem cell properties and 
regulation, as well as for their potential use in regenerative medicine. The common approach to locate stem cell niches has been based on the different expression of a protein marker, or more usually a complex set of protein markers, in stem cell environments compared with more differentiated compartments, as well as on the general property that stem cells are long-term residents of a tissue and have a low proliferative rate (i.e., label-retaining techniques) (Fuchs et al. 2004; Moore and Lemischka 2006). These approaches, although successful, are limited because each type of stem cell niche has its own specific set of markers. Here, we reasoned that the relative telomere length of cells within a given tissue may constitute a bona fide indication of the accumulated divisions and differentiation history of each cell. Based on this, the most primitive stem cell compartments (those where the stem cells reside within tissues) should be enriched in cells with long telomeres, whereas more distant locations should show progressively shorter telomeres.

In this manuscript, we describe a novel general property of adult stem cell compartments. In particular, using confocal telomere quantitiative fluorescence in situ hybridization (Q-FISH, referred to here as telomapping), we find gradients of telomere length within tissues, with the longest telomeres mapping to the known stem cell compartments in skin, small intestine, testis, cornea, and brain of the mouse. This constitutes the description of a novel general property of adult stem cell compartments, which in turn may be useful for their identification. Finally, we show telomere shortening with age in different mouse stem cell compartments, demonstrating that telomere shortening occurs in stem cells associated with mouse aging, which in turn may contribute to stem cell dysfunction and organismal aging.

\section{Results}

Cells with the longest telomeres are enriched at the hair follicle stem cell compartment and show stem cell behavior upon treatment with mitogenic stimuli

To evaluate whether telomere length could be used as a general marker to anatomically map stem cell compartments within adult tissues, we performed confocal telomere FISH (confocal Q-FISH) directly on tissue sections coupled to a single-cell high-throughput Metamorph image analysis platform ("telomapping") (see Materials and Methods). First, we generated single-cell topographic telomere length maps for tail skin sections from 2-moold wild-type mice of a C57BL6 genetic background, which we subdivided into four different epidermal compartments: the hair follicle bulge where the hair follicle stem cell niche is located (Cotsarelis et al. 1990; Oshima et al. 2001; Morris et al. 2004; Tumbar et al. 2004), the hair follicle bulb and the infundibulum where the transitamplifying (TA) cells reside, and the interfollicular epidermis (Fig. 1A,B; Supplemental Fig. S1a). In "resting" untreated wild-type mouse skin, we observed that cells with the longest telomeres, 1800-3000 arbitrary units (a.u.) of telomere fluorescence (red color in Fig. 1A; see Materials and Methods for criteria to establish telomere length ranges within a given tissue) were enriched at the hair bulge, coinciding with the known stem cell niche (Cotsarelis et al. 1990; Oshima et al. 2001; Morris et al. 2004; Tumbar et al. 2004). Immunostaining with the hair follicle stem cell markers CD34 and keratin 15 (K15), as well as the accumulation of label-retaining cells (LRCs) and cells with low proliferation rates (negative Ki67 staining), further confirmed that the longest telomeres localized to the hair bulge (Fig. 3A,B, below; Supplemental Fig. S1b,c; see also Supplemental Fig. S9a,b for CD34 and GFP staining in K15-EGFP mice). These results were confirmed in age-matched mice of a FVB background (Supplemental Fig. S2a,b). These findings indicate that telomeres are progressively shorter as cells move out from the stem cell compartment to the adjacent TA compartments (hair bulb and infundibulum), with the more differentiated layers of the interfollicular epidermis showing the shortest telomeres, in agreement with their longer proliferative and differentiation history. Of note, these differences in telomere length are unlikely to be due to differences in "probe accessibility" or ploidy between different skin compartments as we did not find significant differences between compartments when performing Q-FISH with a centromeric major satellite probe (Supplemental Fig. S3a; Materials and Methods). Furthermore, telomere length differences are not likely to be due to differences in nuclei size between different skin compartments as telomere length is captured for the entire nucleus using the DAPI image (Materials and Methods). Indeed, we did not find major differences in nuclear size between the stem cell compartment (hair bulge) and the interfollicular epidermis and infundibulum compartments that could account for the observed differences in telomere length (Supplemental Fig. S3b). Next, we validated the skin telomapping results using the conventional quantitative telomere FISH technique (Q-FISH) on tissue sections (Materials and Methods). Q-FISH on tissue sections has been extensively used to obtain quantitative and accurate telomere length determinations both in mouse (Gonzalez-Suarez et al. 2000; Muñoz et al. 2005) and human cells (Meeker et al. 2002; 2004; Meeker and De Marzo 2004). Supplemental Figure S4a shows a similar decrease in telomere length when comparing the hair bulge compartment with other skin compartments using telomapping or Q-FISH on tissue sections. Furthermore, there was a linear correlation in telomere length values obtained by these techniques (Supplemental Fig. S4b). In order to calibrate the telomapping technique and to convert fluorescence values into kilobases, we performed telomapping on a paraffin-embedded array of human and mouse cell lines of previously known telomere lengths (Canela et al. 2007; Materials and Methods). Telomapping was able to detect differences in telomere length of $<1 \mathrm{~kb}$ (see comparisons between HeLa and HeLa2 cell lines, and between HeLaS3 and 293T cell lines; $P<0.001$ for both comparisons; Supplemental Fig. S5a). Furthermore, there was a linear correlation between 
Flores et al.

Figure 1. Cells with the longest telomeres are enriched at the hair follicle stem cell compartment and show stem cell behavior upon treatment with mitogenic stimuli. Representative telomere length pseudocolor images of "resting" wild-type $(A)$ and G1 $\operatorname{Terc}^{-/-}(B)$ tail skin. Nuclei are colored according to their average telomere fluorescence in arbitrary units. The different epidermal compartments are indicated and separated from the dermis by a dashed line. Asterisk indicates the sebaceous glands. Bars, $50 \mu \mathrm{m}$. Note the specific enrichment of cells with the longest telomeres at the hair bulge area in both wildtype and Terc $^{-/-}$mice. Bottom panels show the percentage of cells showing a given telomere fluorescence within the indicated epidermal compartment. A total of three skin sections per mouse out of two mice per genotype were used for quantification of percentage of cells and standard deviation. (n) Total number of cells within the indicated compartment used for the analysis. (C) Upon TPA treatment, wild-type epidermal cells showing the longest telomeres (red color) localized not only to the hair bulge but also to the TA compartments (hair bulb and infundibulum). This enrichment of cell with long telomeres to the TA compartments was abolished in TPA-treated G1 $\mathrm{Terc}^{-/-}$skin. (D) Absolute number of cells showing an average telomere fluorescence between 1800 and 3000 a.u. per indicated skin compartment \pm SD. A total of three skin sections per mouse out of a total of two mice per genotype were used for quantification of the number of cells per skin compartment and standard deviation. $(n)$ Total number of cells of each compartment used for the analysis (six independent hair follicle images were counted). (E) Absolute total number of epidermal cells per skin section with telomere fluorescence between 1800 and 3000 a.u. \pm SD. Note that TPA induces a net telomere elongation in wild-type epidermis, which is abolished in G1 Terc $^{-/-}$skin. A total of six skin sections per genotype and condition were used for quantification purposes. (n) Total number of cells in the epidermis included for the analysis. Statistical significance is indicated on top of the bars.

telomapping results and Q-FISH telomere length results as determined by conventional Q-FISH on metaphases (Supplemental Fig. S5b). Finally, using this calibration
A

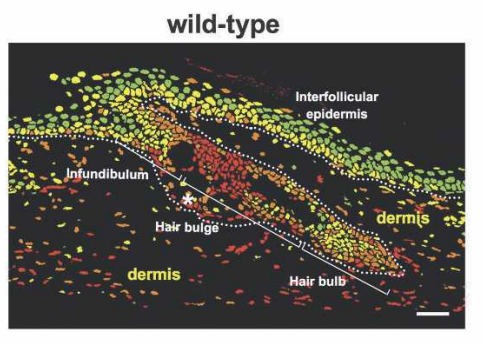

B
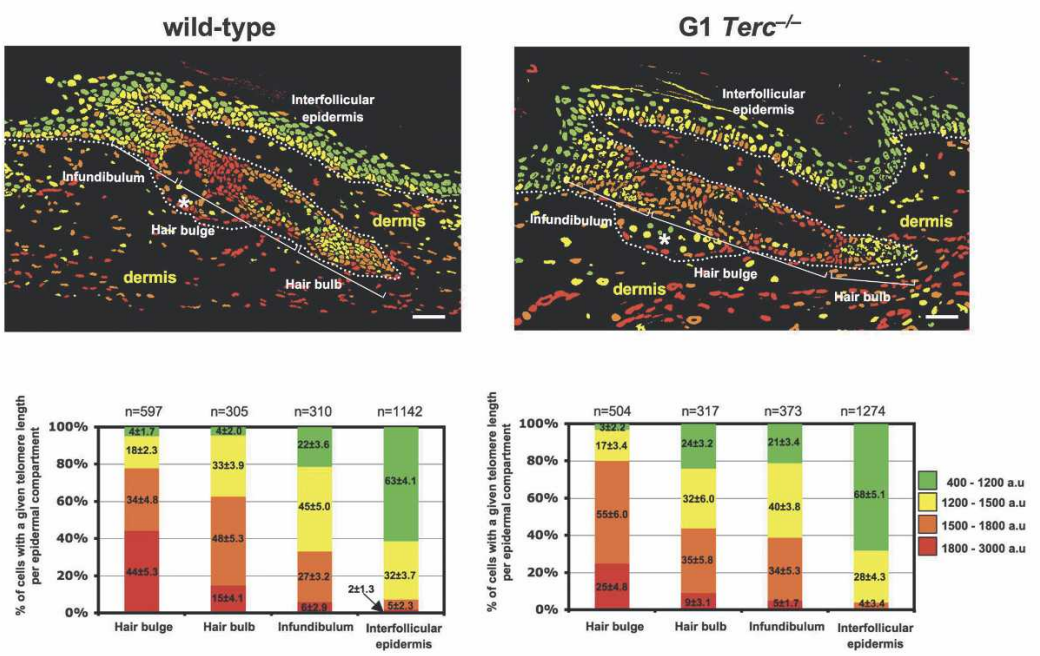

C

TPA-treated skin
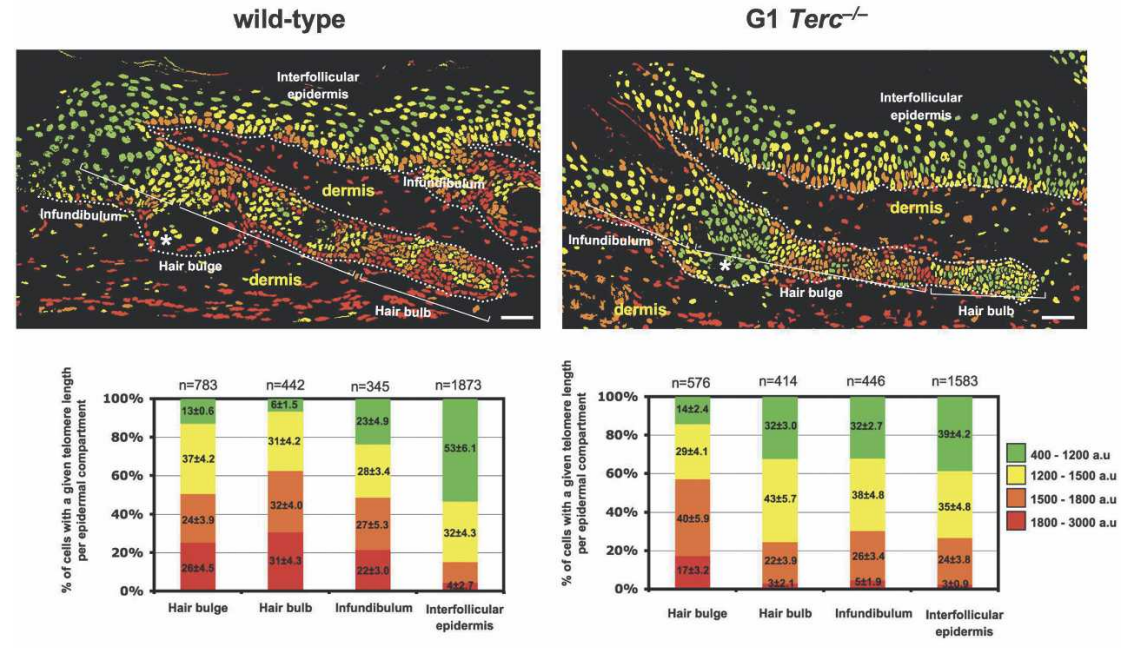

D

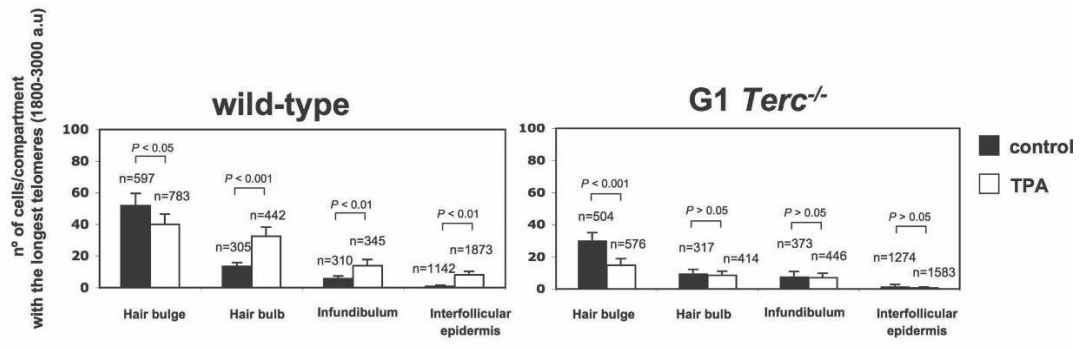

E

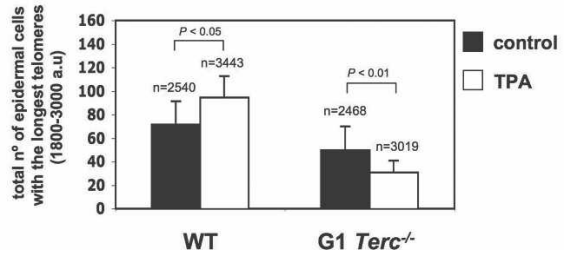

we detect a decrease of telomere length between the hair bulge (stem cell compartment) and the TA compartments of $1.3 \mathrm{~kb}$ (hair bulb) and $3.5 \mathrm{~kb}$ (infundibulum) and 
of $9.8 \mathrm{~kb}$ when comparing the hair bulge with the interfollicular epidermis (Supplemental Fig. S5c).

To further test whether the longest telomeres map to stem cell compartments and to prevent the influence of possible telomerase activation on telomere length, we generated topographic telomere length maps from firstgeneration telomerase-deficient G1 $\mathrm{Terc}^{-/-}$mice (Fig. 1B; Blasco et al. 1997; Ramirez et al. 1997). Similarly to wildtype skin, G1 $\mathrm{Terc}^{-/-}$skin showed an enrichment of cells with the longest telomeres (1800-3000 a.u. of telomere fluorescence) in the bulge area of the hair follicle with the shortest telomeres at the interfollicular epidermis (Fig. 1B), thus confirming that the longest telomeres are enriched at the stem cell compartment. However, the percentage of cells with 1800-3000 a.u. of telomere fluorescence was lower in all skin compartments compared with control wild-type mice, in agreement with the fact that G1 $\mathrm{Terc}^{-1-}$ mice lack telomerase activity (Fig. 1B). Similarly, average telomere fluorescence was lower in G1 $\mathrm{Terc}^{-/-}$mice compared with wild-type mice in all skin compartments (Supplemental Fig. S6). These results demonstrate that the cells with the longest telomeres are enriched at the hair follicle stem cell compartment, while the cells with the shortest telomeres are located in the outer skin layers, indicating that telomeres are shorter as cells go from the more primitive to the more differentiated skin compartments. Furthermore, these results indicate that telomerase activity is important to maintain the overall telomere length of different skin compartments in the mouse, as first-generation telomerase-deficient G1 $\mathrm{Terc}^{-/-}$showed a marked decrease in telomere length compared with age-matched wild-type controls in all skin compartments (see also Fig. 5B, below for telomere shortening with increasing mouse age).

Both telomere length and the catalytic component of telomerase, Tert, have been shown to be critical determinants in the mobilization of hair follicle stem cells out of their niches following treatment with the mitogen TPA (Flores et al. 2005; Sarin et al. 2005). To address whether cells with the longest telomeres within the hair follicles also show characteristic stem cell behavior, we treated wild-type and G1 $\mathrm{Terc}^{-/-}$mice with the mitogenic stimulus TPA, which triggers migration and proliferation ("mobilization") of stem cells out of the niches into the TA compartments (see Supplemental Fig. S7a,b to visualize "migration" of hair bulge cells to the TA compartments upon TPA treatment; see also Flores et al. 2005). Wild-type TPA-treated skin showed a decrease in the percentage of the cells with the longest telomeres at the hair bulge with an accumulation of these cells to the TA compartments (hair bulb and infundibulum), coinciding with an enlargement of these compartments and thickening of the interfollicular epidermis (Fig. 1, cf. C and A). As a consequence, the absolute number of cells containing the longest telomeres decreased at the hair bulge and concomitantly increased in the other epidermal compartments (significant in all cases) (Fig. 1D). Furthermore, the total number of epidermal cells showing 1800-3000 a.u. of telomere fluorescence significantly increased in TPA-treated skin compared with the un- treated wild-type skin $(P<0.05)($ Fig. $1 \mathrm{E})$, suggesting net telomere elongation associated with TPA-induced proliferation in TA compartments (Fig. 1E). Telomere length histograms also showed decreased frequency of long telomeres in hair bulge cells upon TPA treatment, which was concomitant with increased telomere length in cells located at the TA compartment and the interfollicular epidermis (Supplemental Fig. S6). Altogether, these results suggest that upon TPA treatment, wildtype stem cells migrate out of their niche and proliferate in the TA compartments, coincidental with net telomere elongation in these compartments.

To address whether this was dependent on telomerase activity, we studied TPA-treated G1 $\mathrm{Terc}^{-/-}$skin. Similarly to TPA-treated wild-type skin, G1 $\mathrm{Terc}^{-/-}$skin showed a reduction of the percentage of cells with the longest telomeres at the hair bulge (Fig. 1C) coincidental with an enlargement of the TA compartments (Fig. 1, cf. $\mathrm{C}$ and $\mathrm{B}$ ), suggesting that these cells mobilized and proliferated in response to TPA. However, in contrast to TPA-treated wild-type skin, G1 $\mathrm{Terc}^{-/-}$TPA-treated skin did not show increased percentage of cells with the longest telomeres at the TA compartments and the interfollicular epidermis (Fig. 1C,D). Indeed, the total number of epidermal cells showing the longest telomeres was decreased in G1 $\mathrm{Terc}^{-/-}$TPA-treated skin compared with untreated skin $(P<0.01)$ (Fig. 1E), suggesting telomere shortening as the result of TPA-induced proliferation in the absence of telomerase activity. In agreement with this, telomere length histograms of TPA-treated G1 $\mathrm{Terc}^{-/-}$mice showed a decreased frequency of long telomeres in all hair follicle compartments (Supplemental Fig. S6). These results indicate that telomerase actively participates in telomere maintenance upon migration and proliferation (mobilization) of stem cells in response to TPA treatment; in contrast, mobilization of stem cells in the absence of telomerase results in telomere shortening in all skin compartments in G1 $\mathrm{Terc}^{-/-}$mice.

\section{Isolated hair bulge cells from K15-EGFP mice show the longest telomeres}

The results described above indicate that cells with the longest telomeres are enriched at stem cell compartments. To further address this, we set out to determine whether purified skin hair bulge cells (hair follicle stem cell compartment) also showed the longest telomeres. For this, we used K15-EGFP transgenic mice, in which the K15-expressing hair bulge cells are identified by positive GFP expression (Morris et al. 2004; Materials and Methods). GFP-positive cells from these mice have been shown previously to have stem cell properties and to contribute to some aspects of skin regeneration (i.e., wound healing) as well as to have a higher in vitro clonogenic potential than GFP-negative cells (Morris et al. 2004; Ito et al. 2005). To this end, we sorted K15-EGFP skin keratinocytes into GFP-negative $\left(\mathrm{GFP}^{-}\right)$and GFPpositive $\left(\mathrm{GPF}^{+}\right)$cell populations (Materials and Methods). In agreement with previously published data, we recovered $\sim 4 \% \mathrm{GFP}^{+}$cells from total K15-EGFP tail epi- 
dermis, reflecting the relatively low abundance of hair bulge cells with stem cell properties (Morris et al. 2004; Ito et al. 2005). As a control for enrichment in stem cells, we performed in vitro clonogenic assays, where individual colonies are proposed to derive from single stem cells (Flores et al. 2005). Purified GFP ${ }^{+}$cells formed three times more colonies than $\mathrm{GFP}^{-}$cells in clonogenic assays, in agreement with the notion that they are enriched in hair bulge stem cells (Supplemental Fig. S8a; Morris et al. 2004; Ito et al. 2005). Moreover, $\mathrm{GFP}^{+}$cells were more abundant in the larger colonies when using total unsorted K15-EGFP keratinocytes, also supporting the notion that $\mathrm{K} 15-\mathrm{GFP}^{+}$cells are enriched in stem cells (Supplemental Fig. S8b). Importantly, using conventional Q-FISH on cytospin-plated interphasic cells (Materials and Methods), we found that the $\mathrm{GFP}^{+}$keratinocytes showed longer telomeres than the $\mathrm{GFP}^{-}$kera- tinocytes (highly significant, $P<0.001$ ) (Fig. 2A,B). To rule out possible differences in ploidy, we quantified the number of telomere signals per nuclei and found no significant differences between sorted $\mathrm{GFP}^{+}$and $\mathrm{GFP}^{-}$cells $(P=0.457)$ (Fig. 2C). In addition, we ruled out that differences in telomere length could be due to differential "probe accessibility" by performing Q-FISH with a centromeric major satellite probe as control (Fig. 2D,E; Materials and Methods). The decline in telomere length between $\mathrm{GFP}^{+}$and $\mathrm{GFP}^{-}$cells was validated using an independent quantitative telomere FISH technique based on flow cytometry known as Flow-FISH (Materials and Methods). Two mouse cell lines of known telomere length were also included in the Flow-FISH analysis in order to convert telomere fluorescence values into kilobases (Materials and Methods). Again, purified K15$\mathrm{EGFP}^{+}$cells showed significantly longer telomeres than
Figure 2. Isolated hair bulge stem cells from K15-EGFP mice show the longest telomeres and telomerase activity. (A) Representative DAPI and Cy3 images of $\mathrm{GFP}^{+}$ and $\mathrm{GFP}^{-}$FACS-sorted keratinocytes from K15-EGFP mice. (B) Q-FISH histograms showing telomere fluorescence frequencies. Average telomere fluorescence and SD are indicated. Differences in telomere length between $\mathrm{GPF}^{+}$and $\mathrm{GFP}^{-}$cells were highly significant $(P<0.001)$. (n) Number of nuclei used for the Q-FISH analysis from two independent K15-EGFP mice. The red lines highlight the increased frequency of long telomeres in GFP-positive cells. (C) Number of telomere spots per nuclei in sorted $\mathrm{GFP}^{+}$ and $\mathrm{GFP}^{-}$cells indicates similar ploidy. $(D)$ Representative DAPI and Cy3 centromeric images of $\mathrm{GFP}^{+}$and $\mathrm{GFP}^{-}$FACS-sorted keratinocytes from K15-EGFP mice. (E) Quantification of major satellite fluorescence signal in sorted $\mathrm{GFP}^{+}$and $\mathrm{GFP}^{-}$cells. No significant differences in centromere fluorescence were detected between both populations. Two independent mice were used for the analysis. (n) Total number of nuclei used for the analysis. (F) Average telomere length in kilobases of purified GFP-positive and GFP-negative cells from 0.5- and 1.5-yrold K15-EGFP mice as determined by FlowFISH. Bars indicate standard errors. ( $n$ ) Cells analyzed per condition. Statistical significance using Kolmogorov-Smirnov tests is indicated. (G) Telomere length as determined by TRF in sorted $\mathrm{GFP}^{-}$and $\mathrm{GFP}^{+}$tail skin keratinocytes from K15-EGFP mice. Note increased TRF size in $\mathrm{GFP}^{+}$hair bulge cells compared with $\mathrm{GFP}^{-}$cells. $(H)$ Telomerase TRAP activity of purified $\mathrm{GFP}^{+}$and $\mathrm{GFP}^{-}$keratinocytes from K15-EGFP mice. The protein concentration is indicated. Samples were pretreated $(+)$ or not $(-)$ with RNase. (IC) Internal control. HeLa cells are shown as positive control.
A

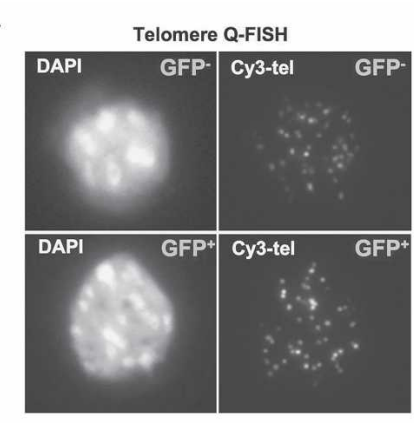

B
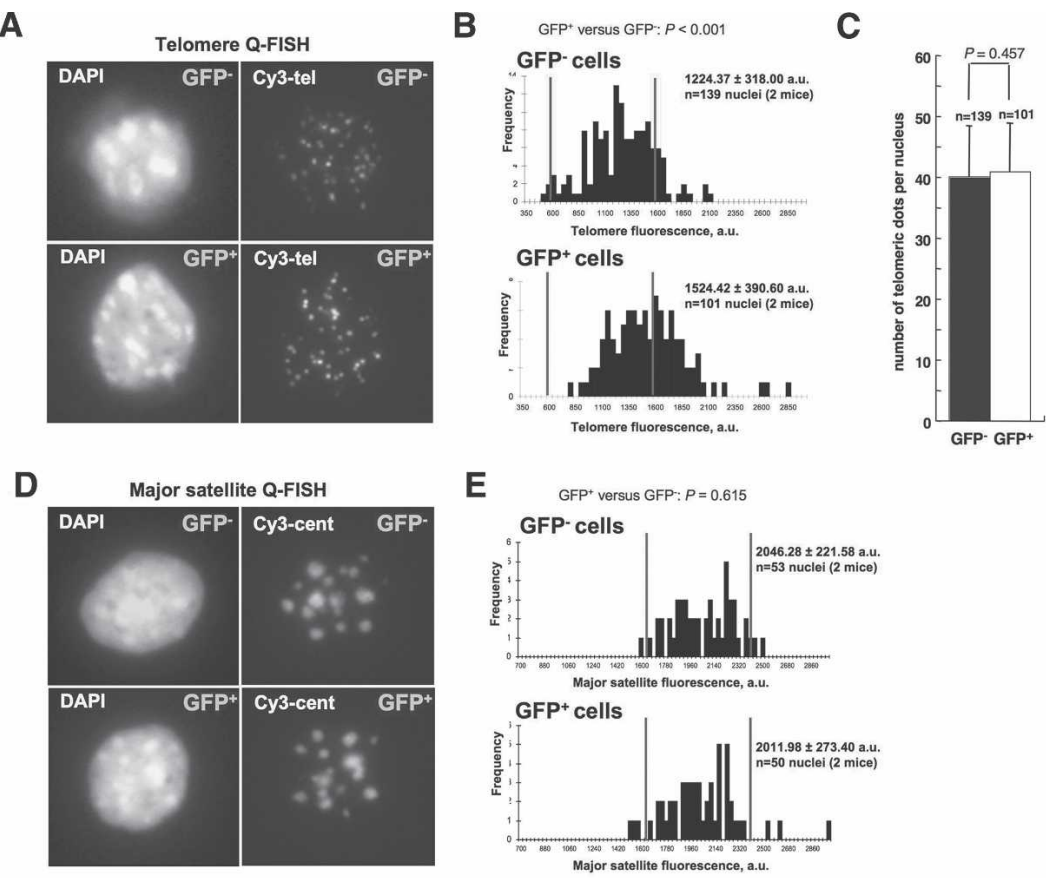

E
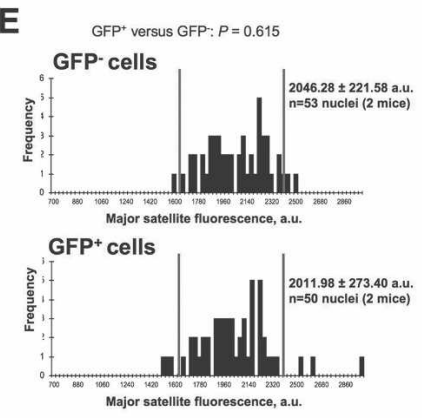

$F$

G
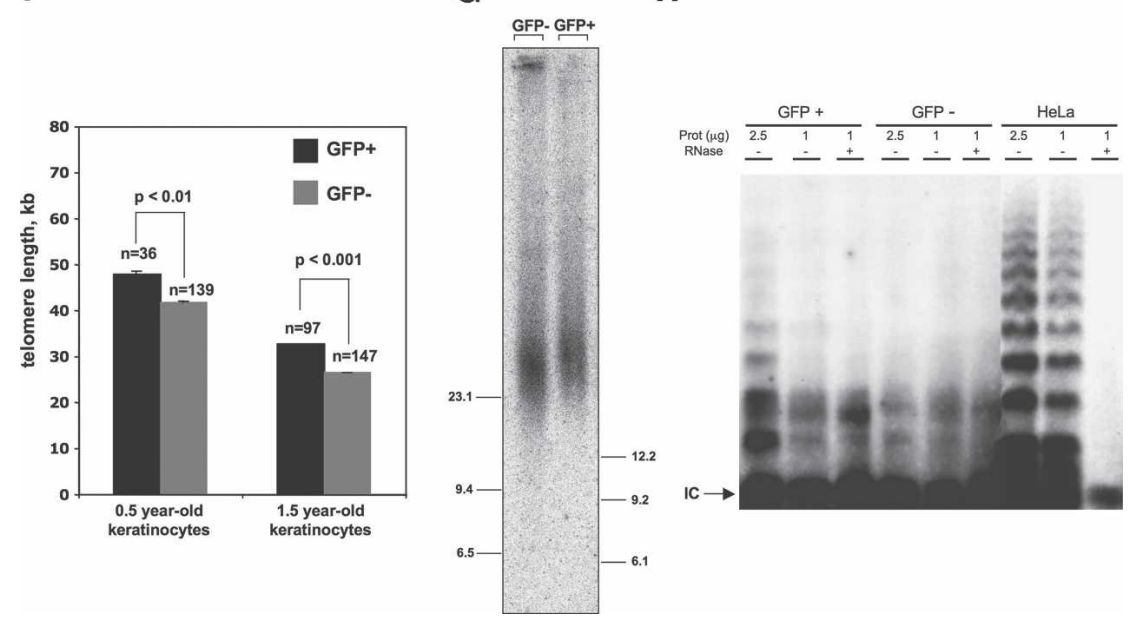
K15-EGFP ${ }^{-}$cells both in young (0.5-yr-old) and old (1.5yr-old) mice $(P<0.01)$ (Fig. $2 \mathrm{~F})$. We estimated a telomere shortening of $6 \mathrm{~kb}$ between K15-EGFP ${ }^{+}$hair bulge keratinocytes (a population enriched in stem cells) and K15$\mathrm{EGFP}^{-}$keratinocytes (a population enriched in differentiated cells) (Fig. 2F), which corresponds to an $\sim 16 \%$ decline in telomere length. Finally, longer telomeres in $\mathrm{K}_{15} \mathrm{EGFP}^{+}$hair bulge cells were also confirmed when using a Southern blot-based technique known as "telomere restriction analysis" (TRF), which is not based on fluorescence (Fig. 2G; Materials and Methods). Of interest, concomitantly with the decreased telomere length in the differentiated skin compartments, we also observed a reduction in telomerase activity when comparing $\mathrm{K} 15-\mathrm{EGFP}^{+}$hair bulge keratinocytes and K15-EGFP ${ }^{-}$ keratinocytes (Fig. 2H), which may contribute to the observed telomere attrition associated with differentiation (see also Fig. 1; Supplemental Figs. S4, S5). Furthermore, the fact that the K15-EGFP ${ }^{+}$hair bulge keratinocytes have telomerase activity, together with the fact that hair bulge cells are slow-cycling cells (negative Ki67 staining in Supplemental Fig. S1b,c) may contribute to their enrichment in longer telomeres.

Next, we combined GFP immunofluorescence with confocal telomere Q-FISH directly on back skin sections from K15-EGFP mice to address whether the $\mathrm{GFP}^{+}$cells within the hair follicle colocalized with the longest telomeres on skin histological sections (Materials and Methods). We first colocalized GFP expression with the CD34 hair bulge marker both in these mice, supporting K15GFP expression in a fraction of the hair bulge CD34positive cells both in tail and back skin (Supplemental Fig. S9a,b; Morris et al. 2004). As shown in Figure 3A, $\mathrm{GFP}^{+}$cells localized to the hair follicle bulge and showed the longest telomeres compared with the $\mathrm{GFP}^{-}$cells (highly significant, $P<0.001$ ) (Fig. 3A,B). Telomapping of $\mathrm{GFP}^{+}$and $\mathrm{GFP}^{-}$back skin cells also indicated a $17 \%$ decrease in telomere length between both compartments, similarly to the decrease obtained by Flow-FISH (see above). In addition, we calculated that $59.3 \%$ of the cells with the longest telomeres (Fig. 3C, red color after telomapping) were $\mathrm{GFP}^{+}$, while this percentage dropped to

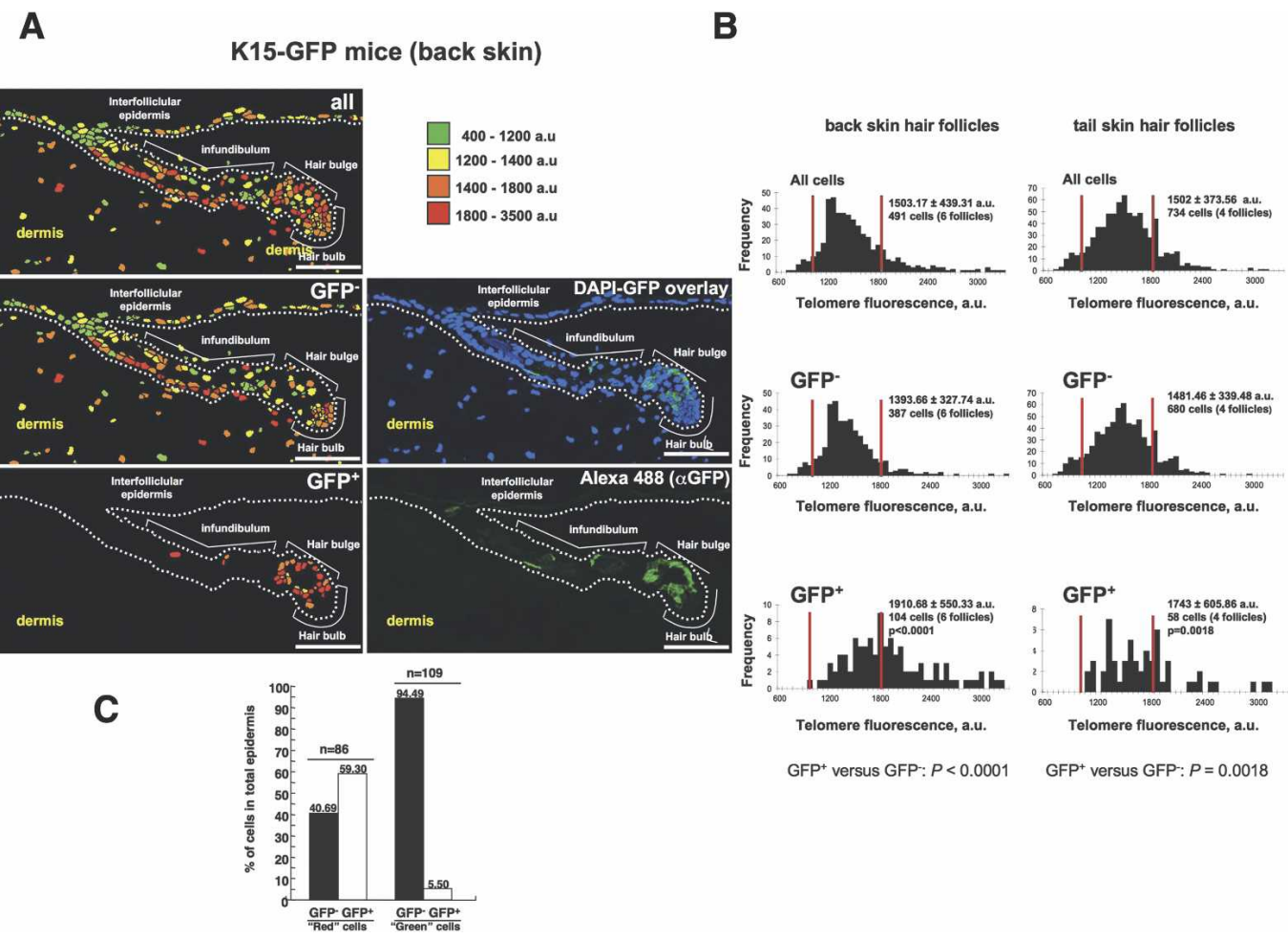

Figure 3. Telomapping maps the longest telomeres to the EGFP ${ }^{+}$cells in K15-EGFP skin sections. (A) Simultaneous detection of GFP and telomere fluorescence in K15-EGFP back skin. The different epidermal compartments are indicated and separated from the dermis by a dashed line. Right panels show confocal images corresponding to Alexa488 fluorescence and the combined DAPI + GFP image. Note that GFP-expressing cells localize to the bulge area of the hair follicle. Left panels show topographic telomere length maps generated according to GFP status: all nuclei, GFP ${ }^{-}$nuclei, and $\mathrm{GFP}^{+}$nuclei. Nuclei are colored according to their average telomere fluorescence in arbitrary units. GFP-positive cells at the hair bulge showed the longest telomeres. Bars, $50 \mu \mathrm{m}$. $(B)$ Telomere fluorescence frequency histograms according to GFP status in back and tail skin hair follicles from K15-EGFP mice. Differences in telomere length between $\mathrm{GPF}^{+}$and $\mathrm{GFP}^{-}$cells were highly significant $(P<0.001)$, both in the back and tail skin. Average telomere fluorescence and SD are indicated. (n) Number of nuclei analyzed. Four to six skin sections of either tail or back skin from a total of two mice were analyzed. The red lines highlight the increased frequency of long telomeres in GFP-positive cells. $(C)$ Percentage of cells with the longest telomeres (red color) or with the shortest telomeres (green color) that are either $\mathrm{GFP}^{+}$or $\mathrm{GFP}^{-}$. Note that $\mathrm{GFP}^{+}$cells are enriched in the population of the cells with the longest telomeres. 
$5.5 \%$ in cells with the shortest telomeres (Fig. 3C, green after telomapping). Altogether, these results suggest that $>50 \%$ of epidermal cells with the longest telomeres are K15-expressing hair bulge cells $\left(\mathrm{GFP}^{+}\right.$cells), which in turn have been shown to be enriched in stem cells (Morris et al. 2004; Ito et al. 2005; see also Supplemental Fig. S8).

The longest telomeres are a general feature of different mouse stem cell compartments (small intestine, cornea, testis, brain)

Altogether, the above-described findings suggest that the most primitive compartments within the skin are characterized by having cells with the longest telomeres compared with the more differentiated compartments. To generalize and test this hypothesis in other tissues besides the skin, we performed telomapping in histological sections from small intestine, cornea, testis, and brain, where the corresponding stem cell compartments have been well characterized in the mouse. In mice, a small intestine stem cell niche has been localized to the bottom of the intestinal crypts at approximately the +4 position, right above the Paneth cells (positions +1 to +3 ) and below the TA compartment (position greater than +5 ), whereas the most differentiated cells are located at the intestinal villi (see scheme in Fig. 4B; Supplemental Fig. S10a shows known markers of Paneth cells [lysozyme] and stem/TA compartments [Sox9]; Marshman et al. 2002; Gregorieff et al. 2005). Topographic telomere length maps of small intestine histological sections localized the highest percentage of cells with the long telomeres (1700-3000 a.u. of telomere fluorescence) (see Materials and Methods for criteria on telomere length ranges) above the Paneth cells and in the TA compartment (Fig. 4A,C), although scattered cells with long telomeres are also seen at the crypt base. Interestingly, Barker et al. (2007) recently identified Lgr5-positive crypt base columnar cells located interspersed between Paneth cells as putative stem cells for the small intestine. Next, we generated histological telomere length maps for mouse cornea and testis, two other epithelial tissues where the SC compartment has been spatially defined. Corneal stem cells reside at the limbus, the peripheral zone of the cornea lying above the ciliary body that stains positive for the keratin19 marker (Supplemental Fig. S10b; Lehrer et al. 1998; Lavker et al. 2004). From this location, corneal stem cells migrate toward the central corneal epithelium as their differentiation program proceeds (Lehrer et al. 1998). Telomapping of eye sections revealed that an average of $50 \%$ of the limbal cells possess the longest telomeres (1400-3000 a.u. of telomere fluorescence) (Materials and Methods), a percentage that gradually diminishes as cells move centripetally toward the center of the cornea (Fig. 4E). The percentage of cells with the longest telomeres further increased to $68 \%$ within the limbal basal layer (see Figure 4E, inset), a compartment where corneal SC are particularly enriched (Lehrer et al. 1998). Comparison of average telomere fluorescence between the limbus and the central cornea further indicated that the corneal stem cell compartment harbors the cells with the longest telomeres $(P<0.001)$ (Fig. 4E).

In mouse testis, spermatogenesis starts at the periphery of the seminiferous tubules, where the germ stem cells reside (Guan et al. 2006; Supplemental Fig. S10c shows Sox9 and vimentin stainings, known markers of the peripheral cells). By a series of mitotic divisions followed by meiosis, male germ stem cells give rise sequentially to spermatogonia, spermatocyte, spermatid, and spermatozoa as they move to a more luminal position (Brinster 2002). Telomapping of testis sections showed that the periphery (first and second layers) of the seminiferous tubules presented the highest percentage of cells with long telomeres (1800-3000 a.u of telomere fluorescence) (Fig. 4F; Materials and Methods). In contrast, the lumen zone is highly enriched with cells showing the shortest telomeres. Comparisons of telomere fluorescence frequency histograms of the periphery and the lumen areas also indicate a decreased telomere length in the lumen compared with the periphery $(P<0.001)$ (Fig. 4F), reflecting their differentiation program. Again, these differences in telomere length are unlikely to be due to differences in "probe accessibility" or ploidy between different testis compartments as we did not find significant differences when performing Q-FISH with a centromeric major satellite probe (Supplemental Fig. S3c; Materials and Methods).

Finally, we analyzed by telomapping coronal sections of the adult mouse brain containing areas of neurogenesis. To date, two different spatial stem cell niches have been characterized in the adult mouse brain: the subventricular zone (SVZ) of the lateral ventricule and the subgranular zone (SGZ) at the hippocampus (Gage 2000; Alvarez-Buylla and Lim 2004). At the hippocampus, neural stem cells lie in the SGZ, an area located between the granular cell layer (GCL) and the hilus $(\mathrm{H})$ that contain Sox2-positive cells (Supplemental Fig. S10d). From their basal position, neural stem cells proliferate, migrate, and differentiate into the more apical GCL (Gage 2000). Topographic telomere length mapping revealed that cells with the longest telomeres (1400-3000 a.u. of telomere fluorescence) are enriched at the SGZ, showing progressively shorter telomeres as they enter the abutting GCL (Fig. 4G). Average telomere fluorescence and telomere length distributions also indicated longer telomeres at SGZ compared with GCL $(P<0.001$; Fig. 4G), further reflecting that the hippocampus stem cell compartment (SGZ) is enriched in cells having the longest telomeres.

\section{Telomere shortening with age in mouse stem cell compartments}

Next, we used telomapping to address whether telomeres shorten in different mouse stem cell compartments with increasing age, which in turn could contribute to stem cell dysfunction with age. A role for telomere shortening in mouse aging and stem cell aging was suggested previously by the reduction in both median and maximum life span (García-Cao et al. 2006) as well as in 
A

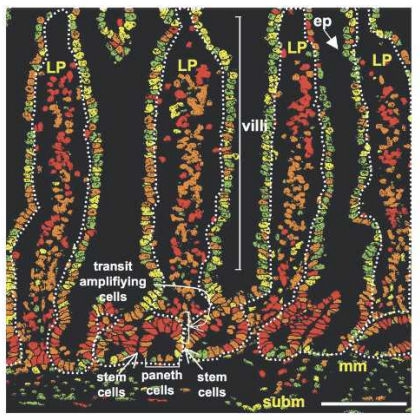

C

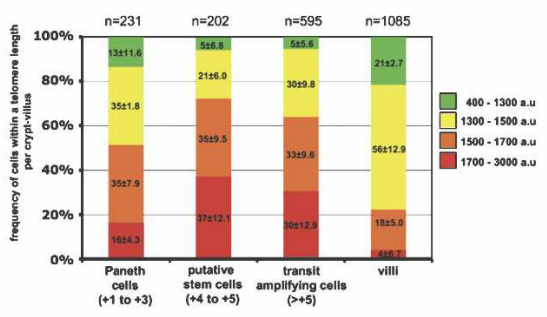

B

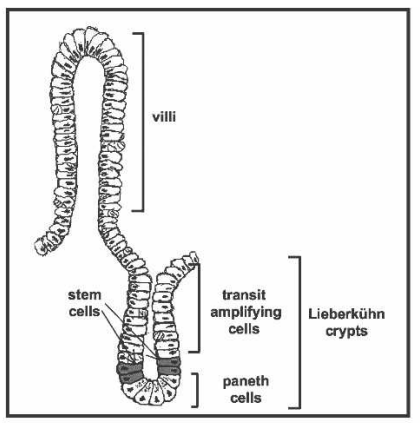

D

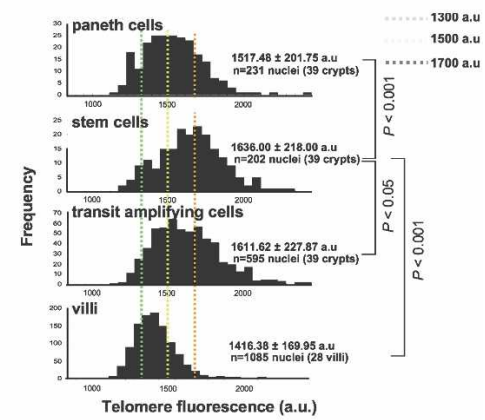

E

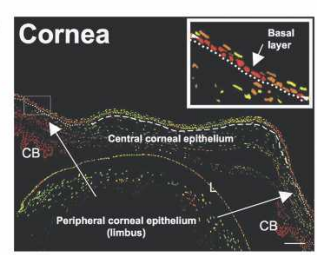

F

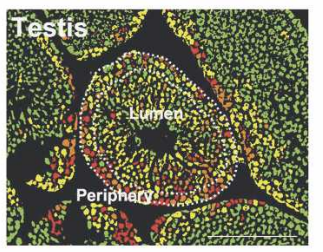

G

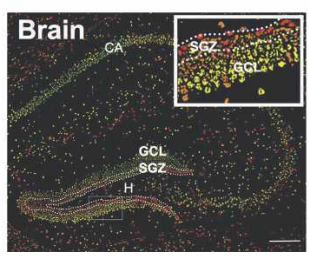

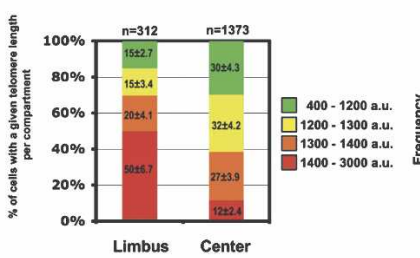

Limbus Center
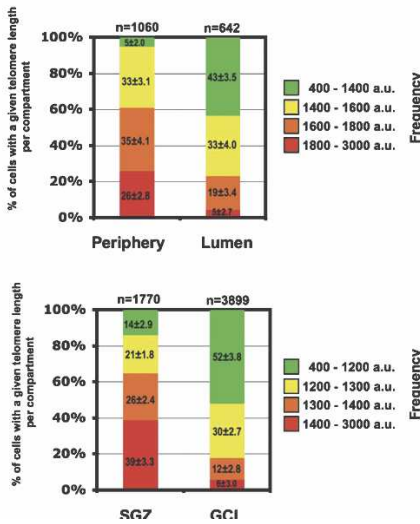

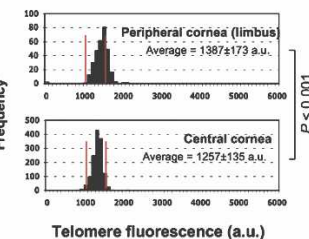

Telomere fluorescence (a.u.)

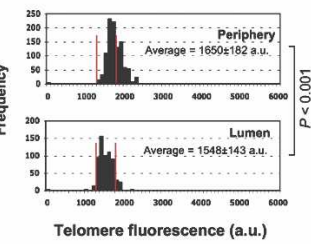

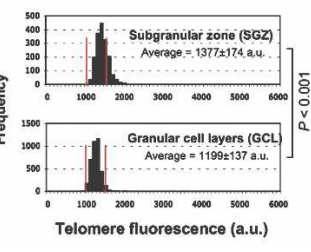

Figure 4. Cells with the longest telomeres locate to stem cell compartments in different origin mouse tissues. (A) Representative telomapping image of a small intestine section generated from confocal telomere QFISH images. The different small intestine compartments are indicated. The dashed line separates the epithelial cells (ep) from other cell types not studied here: lamina propia (LP), muscularis mucosa $(\mathrm{mm})$, and submucosa (subm). Bar, $70 \mu \mathrm{m}$. Nuclei are colored according to their average telomere fluorescence in arbitrary units. (B) Scheme depicting the small intestine villi and Lieberkühn crypts. The crypts are further divided in (1) the Paneth cells at the bottom of the crypt between positions +1 and $+3,(2)$ positions +4 to +5 , and (3) the TA compartment at positions greater than +5 . (C) Percentage of cells showing a given telomere fluorescence within the different compartments. The +4 to +5 positions and the TA compartment are enriched in cells with the longest telomeres (red color), while the villi are enriched in cells with the shortest telomeres (green color). Average and SD are indicated. A total of 39 crypts and 28 villi from three independent mice were quantified. $(n)$ Number of nuclei per compartment analyzed. Number in parentheses indicates the cell position in the crypt. (D) Telomere length frequency histograms for cells located in the indicated compartments. Average and SD are indicated. A total of 39 crypts and 28 villi from three independent mice were quantified. (n) Number of nuclei per compartment analyzed for telomere FISH. Vertical colored lines indicate the different telomere fluorescence ranges. All telomere fluorescence comparisons between the +4 to +5 positions and the rest of the compartments are highly significant $(P<0.001)$, except significant $(P<0.05)$ for comparison with the TA compartment. $(E-G)$ Left panels show representative telomapping images from cornea $(E)$, testis $(F)$, and brain hippocampus $(G)$ of wild-type mice. Nuclei are colored according to their average telomere fluorescence in arbitrary units. Bars, $200 \mu \mathrm{m}$. Magnifications of cornea and brain are also shown. Note that cells with the longest telomeres localize preferentially within the described stem cell compartment of each organ. Middle panels show the percentage of cells containing a given telomere fluorescence within each epidermal compartment. Right panels show telomere fluorescence histograms of nuclei in each compartment. Average telomere fluorescence and SD are indicated. The red lines highlight the increased frequency of cells with long telomeres in the analyzed stem cell compartments. A total of six different images from each organ from three independent mice were used for quantification purposes. (CB) Ciliary body, (L) lens, (SGZ) subgranular zone, (GCL) granular cell layer, (H) hilus, (CA) pyramidal cell layers. All telomere fluorescence comparisons between each of the stem cell compartments and the corresponding differentiated compartment are highly significant $(P<0.001)$.

stem cell functionality (Flores et al. 2005) found in earlygeneration $\mathrm{Terc}^{-/}$mice, which is progressively aggravated with increasing mouse generations concomitant with gradual reduction in telomere length (Flores et al. 2005; García-Cao et al. 2006). Previous studies showed that telomeres shorten with age in some tissues of Mus spretus (Prowse and Greider 1995; Coviello-McLaughlin and Prowse 1997), a mouse species with telomeres similar in length to human telomeres; however, telomere shortening with age in the Mus musculus C57Bl6 laboratory strain has not been reported to date due to very long telomeres in this strain. Telomapping revealed that 

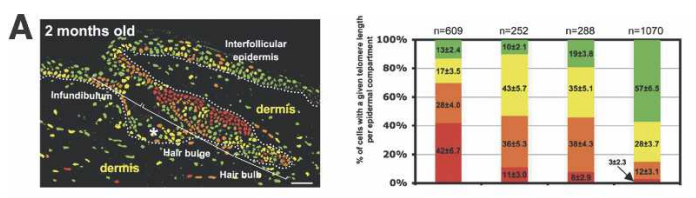

C
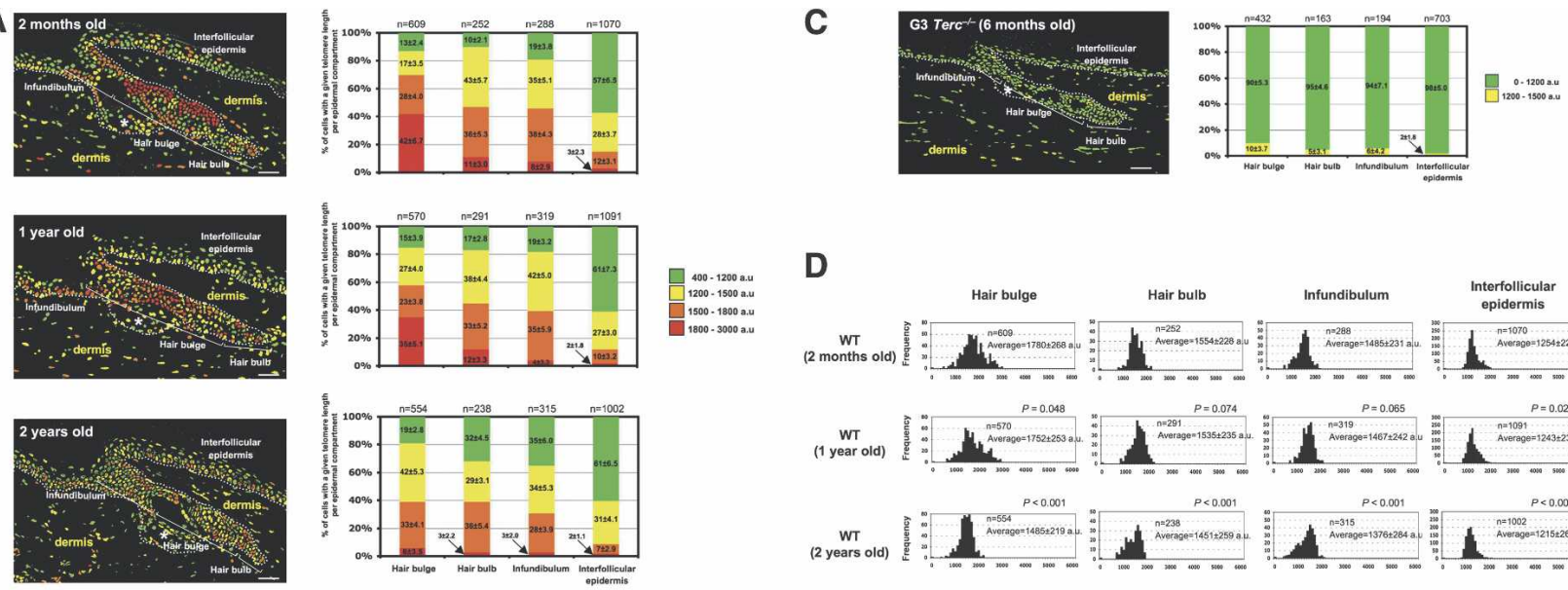

D
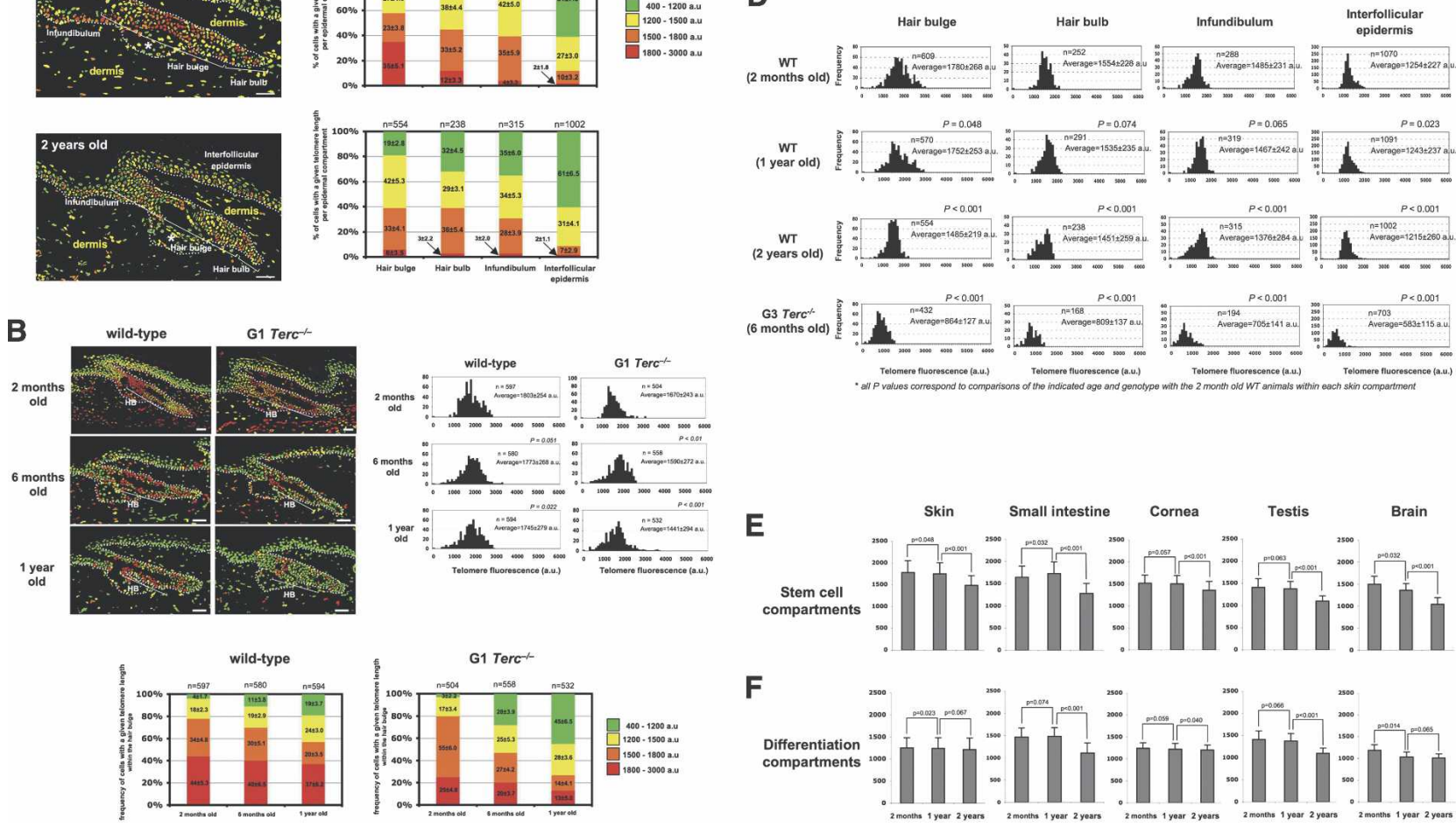

$\mathbf{F}$

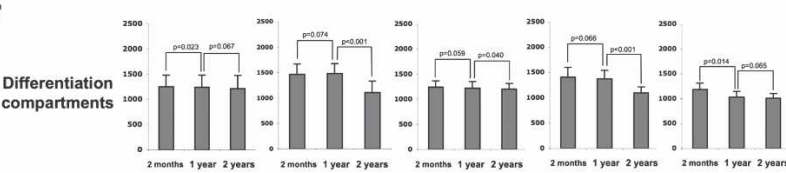

Figure 5. Telomere shortening with age in mouse stem cell compartments. Representative telomapping images of different age wild-type $\left(A, B\right.$, right panels), G1 (B, left panels), and G3 $\mathrm{Terc}^{-/-}(C)$ tail skin. Nuclei are colored according to their average telomere fluorescence in arbitrary units. The different epidermal compartments are indicated and are separated from the dermis by a dashed line. Asterisk indicates the sebaceous glands. Bars, $50 \mu \mathrm{m}$. Note that cells with the longest telomeres at the hair bulge area decrease in both wild-type mice and G1 $\mathrm{Terc}^{-/-}$mice with increasing age, but with a higher rate in the case of G1 Terc $^{-/-}$mice. (B) Right panels show the percentage of cells showing a given telomere fluorescence within the indicated epidermal compartment. A total of two skin sections per mouse out of three mice per genotype were used for quantification of percentage of cells and standard deviation. $(n)$ Total number of cells within the indicated compartment used for the analysis. $(D)$ Telomere length frequency histograms for cells located in the indicated compartments in mice of the indicated age and genotype. A G3 $\mathrm{Terc}^{-/-}$mouse is shown for comparison. (n) Number of nuclei per compartment analyzed for telomere FISH. Statistical significance values are indicated. (E) Average telomere fluorescence in the indicated stem cell compartments at the indicated age. Note a faster rate of telomere loss at $>1 \mathrm{yr}$ of age. $(F)$ Average telomere fluorescence in the indicated differentiated compartments of different tissues at the indicated ages.

both the percentage of cells with the longest telomeres (Fig. 5A), as well as the average telomere length (Fig. 5D), decreased in all skin compartments at $2 \mathrm{yr}$ of age in wildtype C57Bl6 mice. Similarly, average telomere length at the hair bulge cells decreased significantly from $1780 \pm 268$ a.u. to $1485 \pm 219$ a.u. $(P<0.001$; Fig. $5 D)$, demonstrating telomere shortening in hair follicle stem cells at old ages. In parallel, we performed telomapping in the skin of first generation (G1) and third generation (G3) Terc-deficient C57B16 mice. As expected, G1 Tercdeficient mice present a higher rate of telomere loss $(13.7 \%)$ during the first year of life compared with wild type $(3.2 \%)$, in agreement with the lack of telomerase activity on these mice (Fig. 5B), and G3 Terc-deficient mice showed a dramatic reduction of the percentage of cells with the longest telomeres, as well as of average telomere length in all skin compartments (Fig. 5C,D), in agreement with their severe epidermal stem cell dysfunction (Flores et al. 2005). The decreased telomere length with aging in both the stem cell compartment and the more differentiated skin compartments was also confirmed by Flow-FISH analysis using the K15-EGFP reported mouse. In particular, Flow-FISH showed that both sorted $\mathrm{GFP}^{+}$keratinocytes (enriched in stem cells) and $\mathrm{GFP}^{-}$keratinocytes (enriched in differentiated cells) present a reduction of telomere length when comparing 0.5 -yr-old mice with 1.5-yr-old mice (Fig. 2F).

Similarly to skin, we detected telomere shortening in other mouse stem cell compartments including the small intestine, cornea, testis, and brain when compar- 
ing 2-mo-old mice with 2-yr-old mice (Supplemental Figs. S11-S14), further supporting the notion that telomeres shorten at old ages in different stem cell compartments of the mouse, which in turn may result in agerelated stem cell dysfunction. Of note, telomere shortening with age in Mus musculus male germ cells is in agreement with previously reported telomere shortening with age in Mus spretus testis when comparing young (0- to 11-mo-old) and old ( $\geq 12$-mo-old) animals (CovielloMcLaughlin and Prowse 1997). Finally, comparison of telomere shortening with age in all tissues studied here (Fig. 5E,F) indicates that telomere erosion rates in the stem cell compartments vary at different ages, ranging from a slight reduction in length from 2-mo-old to 1-yrold animals to a rapid significant telomere loss when comparing 1-yr-old and 2-yr-old mice (Fig. 5E,F). These results indicate that the mechanisms of telomere length maintenance decline more rapidly at advanced ages, which in turn may contribute to stem cell aging and therefore to aging phenotypes. In this regard, we observed a significant decrease in telomerase activity levels with increasing age in primary skin keratinocytes derived from wild-type mice at different ages (Supplemental Fig. S15), suggesting that loss of telomerase activity with age may be one of the mechanisms to explain telomere attrition associated with aging in the stem cell compartments. These results agree with previous findings where a reduction in telomerase activity with increasing age was found in rat myocytes (Leri et al. 2000).

\section{Reduced clonogenic potential of mouse skin cells with age}

The fact that telomeres shorten with age in different stem cell compartments in the mouse may suggest that the mechanisms for telomere length maintenance decline more rapidly at advanced ages, and that this telomere shortening may contribute to stem cell aging and therefore to aging phenotypes. To address this, we compared the functionality of mouse epidermal stem cells at different ages using clonogenic assays (Materials and Methods), which reflect on the proliferative potential of epidermal stem cells (Flores et al. 2005). In agreement with their shorter telomeres, keratinocytes directly isolated from 27- to 31-mo-old mice formed significantly fewer colonies than those derived from 2-mo-old mice $(P<0.001$; Fig. 6$)$, indicating a decreased clonogenic potential of epidermal cells with aging. These results are in agreement with previous findings in human skin keratinocytes that showed decreased clonogenic potential with increasing age (Barrandon and Green 1987). Since a similar reduction in clonogenic potential is found in telomerase-deficient mice with progressively shorter telomeres (Flores et al. 2005), these findings suggest that telomere erosion associated with normal aging may influence the observed decreased stem cell functionality.

\section{Discussion}

In summary, these findings demonstrate a hierarchical organization of cells of a common origin within tissues according to their telomere length in all organs

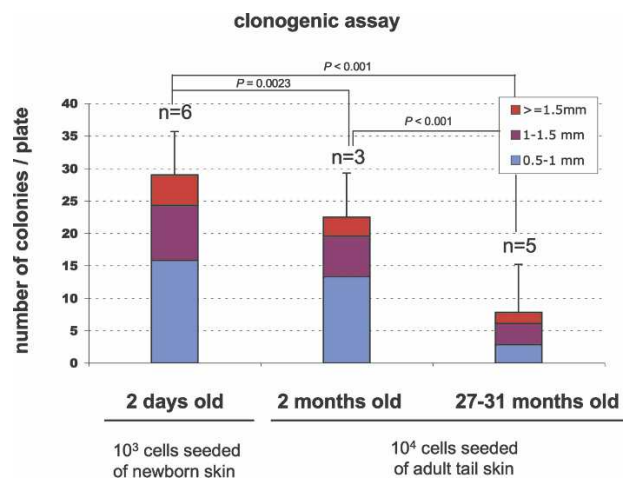

Figure 6. Decreased clonogenic potential of epidermal stem cells with mouse aging. Aging affects the proliferative potential of mouse keratinocyte stem cells. Quantification of size and number of macroscopic colonies obtained from isolated keratinocytes from 2-d-old, 2-mo-old, and 27- to 31-mo-old mice and cultured for $10 \mathrm{~d}$ on J2-3T3 mitomycin C-treated feeder fibroblast. Note that colony number decreases with aging. Statistical significance values are indicated.

examined so far (skin, small intestine, cornea, testis, and brain), with the longest telomeres generally marking the most primitive adult stem cell compartments and the shortest telomeres in the more differentiated compartments within a given tissue. Of note, the rate of telomere shortening within a given tissue appeared larger than expected from mere cell division, which may reflect on active telomere degradation mechanisms associated with differentiation, something that remains unexplored to date. Since cell hierarchies are general features of almost all adult organs, identification of the longest telomeres may represent a novel general marker of adult stem cell compartments, which combined with current ways to identify stem cells such as the so-called "labelretaining techniques" (Cotsarelis et al. 1990; Braun et al. 2003; Flores et al. 2005) may be useful in identifying new stem cell compartments.

Finally, we make the novel finding that Mus musculus telomeres shorten with age in all different stem cell compartments studied here, which in turn may result in decreased stem cell functionality at old age. The correct identification and characterization of adult stem cells in healthy and disease conditions could lead to new clinical applications, such as aid in their isolation as well in the study of their response to different treatments. In particular, tumors have been shown previously to show shorter telomeres compared with the normal surrounding tissue (Meeker et al. 2002, 2004; for review, see Meeker and De Marzo 2004). The ability to identify different cell populations according to their telomere length within tumors will enable researchers to monitor their behavior in response to different stimuli or therapeutic treatments.

\section{Materials and methods}

Mice, treatment regimens, and mouse sample collection

Unless otherwise specified, all mice used were 2-mo-old males from a C57BL6 genetic background. Mice of a FVB genetic back- 
ground ( 2 mo old) were also used in Supplemental Figure S1. $\mathrm{Terc}^{-/}$and K15-EGFP mice were described previously (Blasco et al. 1997; Morris et al. 2004).

To induce epidermal stem cell "mobilization" (activation, migration, and proliferation), tail skin in the telogen (resting) phase of the hair cycle was topically treated every $48 \mathrm{~h}$ with TPA (20 nmol in acetone) for a total of four doses. Control mice of each genotype were treated with acetone alone. Twenty-four hours after the last TPA treatment, mice were sacrificed and the tail skin was analyzed. To assess stem cell migration, a single dose of TPA (20 nmol in acetone) was topically added to K15EGFP back and tail skin.

For telomere length analyses, samples from mouse tail and back skin, small intestine, cornea, testis, and brain were harvested and fixed overnight in neutral-buffered formalin at $4^{\circ} \mathrm{C}$; dehydrated through graded alcohols and xylene; and embedded in paraffin. Prior to embedding, dissected skin was cut parallel to the spine in order to obtain longitudinal hair follicle sections. The intestinal tract was flushed with PBS and rolled up in a compact circle using longitudinally oriented jejunal sections for analysis. For cornea and testis analyses, whole eyes and testis were cut in half prior to dehydration. Finally, brain was coronaldissected to harvest the rostral hippocampus. In all cases, $5 \mu \mathrm{m}$ sections were used for Q-FISH and immunostaining analyses.

\section{Telomerase assay}

Telomerase activity was measured with a modified telomere repeat amplification protocol (TRAP) as described (Blasco et al. 1997). An internal control for PCR efficiency was included (TRAPeze kit Oncor). HeLa cells were included as a positive control for telomerase activity.

\section{Confocal Q-FISH on histological sections}

For Q-FISH, paraffin-embedded tissue sections were hybridized with a PNA-tel Cy3-labeled probe, and telomere length was determined as described (Zijlmans et al. 1997; Gonzalez-Suarez et al. 2000; Samper et al. 2000; Muñoz et al. 2005). DAPI and Cy3 signals were acquired simultaneously into separate channels using a confocal ultraspectral microscope (Leica TCS-SP2A-OBS-UV) using a PL APO $20 \times / 0.70 \mathrm{PH} 2$ lens with Leica LCS software and maximum projections from image stacks $(10 \mathrm{sec}-$ tions at steps of $1.0 \mu \mathrm{m}$ ) were generated for image quantification. The DPSS-561 laser (Cy3 laser) was held at a constant intensity to capture all of the mouse tissue images.

Generation of topographic telomere length maps on histological sections or 'telomapping'

Quantitative image analysis was performed on confocal images using the Metamorph platform (version 6.3r6, Molecular Devices). The DAPI image was used to define the nuclear area and the Cy3 image was used to quantify telomere fluorescence. In all cases, background noise was subtracted from each image prior to quantification. The DAPI images were signal-intensity thresholded, segmented, and converted to 1-bit binary images. Nuclear size was calculated using Metamorph's "Integrated Morphometric analysis" module. The binary DAPI mask was applied to the matching $\mathrm{Cy} 3$ to obtain a combined image with telomere fluorescence information for each nucleus. Cy3 fluorescence intensity (telomere fluorescence) was measured as "average gray value" (total gray value per nuclei area) units (arbitrary units of fluorescence). These "average telomere fluorescence" values always represent the average Cy3 pixel intensity for the total nuclear area, and not the average value of individual telomere spot intensities, therefore ruling out that differences in nuclear size may influence telomere length measurements. A code of four colors was used to classify the nuclei according to their average telomere fluorescence. Telomere fluorescence ranges were initially set up to allocate in each range roughly one-fourth of the total cells of a given tissue in wild-type mice of 2 mo of age. Subsequently, telomere fluorescence ranges were fine-adjusted to better delineate the location of stem cell compartments in different tissues. Telomere fluorescence ranges of a given tissue obtained in this manner were then maintained constant between genotypes, treatment, and ages to facilitate comparisons. Finally, telomere fluorescence values for each histological region were exported to Excel, and the frequency histograms were generated. A macro created using the Metamorph platform allowed the automated and usercontrolled processing of the DAPI and Cy3 images to obtain the telomap images (available upon request).

To avoid differences due to variation in section thickness, we used paraffin slices of the same thickness $(5 \mu \mathrm{m})$ in all the tissues analyzed. Additionally, to avoid possible variations due to different roughnesses of the paraffin on the slices, confocal capture conditions were set to cover the entire fluorescence signal (maximum projections of 10 sections at steps of $1.0 \mu \mathrm{m}$ ). To avoid differences in day-to-day staining efficiency variation, QFISH and immuno-Q-FISH staining as well as image capture were performed for each given tissue in the same day.

Finally, to control for differences in ploidy and probe accessibility, we used a Cy3-labeled PNA probe directed against mouse major satellite repeats $\left(5^{\prime}\right.$-TCGCCATATTCCAGGTC$\left.3^{\prime}\right)$. No significant differences in centromeric fluorescence were detected between different skin and testis compartments (Supplemental Fig. S3a,c), ruling out that differences in "probe accessibility" or ploidy account for the observed differences in telomere length in the skin or the testis. Furthermore, we ruled out that differences in nuclear size between different compartments (i.e., skin) could account for the observed differences in telomere length (Supplemental Fig. S3b). Similarly, no significant differences in centromeric fluorescence were detected between $\mathrm{GFP}^{+}$and $\mathrm{GFP}^{-}$sorted cells from the K15-EGFP mouse model (Fig. 2D,E), again ruling out that differences in "probe accessibility" or ploidy account for the observed differences in telomere length between between $\mathrm{GFP}^{+}$and $\mathrm{GFP}^{-}$cells. To better assess differences in centromeric signal, maximum projections of 16-bit confocal images were obtained from paraffin sections.

Finally, to verify whether image analyses by conventional Q-FISH and telomapping give similar results, the same confocal image of tail skin was quantified using the TFL-TELO program (gift from Dr. Lansdorp, Vancouver) and the Metamorph platform (version 6.3r6, Molecular Devices) (Supplemental Fig. S4).

\section{Labeling of LRCs}

LRCs were obtained as described (Cotsarelis et al. 1990) with some modifications. Briefly, groups of six neonatal mice per genotype were injected with $50 \mathrm{mg}$ of BrdU (Sigma) per kilogram of body weight, diluted in PBS. Each animal received a daily injection beginning at day 4 of life for a total of $5 \mathrm{~d}$. After the labeling period, mice were allowed to grow for $60 \mathrm{~d}$ before the initiation of any treatment. Cells retaining the label at the end of the treatment were identified as LRCs.

\section{Immunohistochemistry}

Skin sections $(5 \mu \mathrm{m})$ were used for immunohistochemistry. Prior to immunohistochemistry, slides were deparaffinated, re- 
hydrated, and immersed in $10 \mathrm{mM}$ citrate solution, and epitopes were retrieved by three high-power, 5-min microwave pulses. Slides were washed in water, blocked in a 1:10 dilution of normal goat serum (Vector Laboratories), and incubated with primary antibodies: CD34 at 1:200 (RAM34, BD Biosciences), keratin 15 at 1:500 (LHK15, NeoMarkers), Ki-67 antibody at 1:200 (SP6, Master Diagnostica), BrdU at 1:35 (Roche), Sox2 at 1:300 (Chemicom), Sox9 at 1:200 (Chemicom), Lysozime at 1:300 (DAKO), Keratin 19 at 1:10 (Development Studies Hybridoma Bank), and Vimentin at 1:25 (Fitzgerald). Slides were then incubated with secondary biotinylated antibodies from Vector Laboratories (goat anti-rabbit at 1:200 or goat anti-mouse at 1:200), followed by signal development with an immunoperoxidase reagent (ABC-HRP, Vector Laboratories) and DAB (Sigma) as the substrate. Sections were lightly counterstained with hematoxylin and analyzed by light microscopy.

\section{Immunofluorescence of GFP and CD34 in K15-EGFP skin}

Samples from K15-EGFP mouse tail and back skin from 2-moold mice were harvested, fixed, deparaffinated, and epitoperetrieved as described above. Slides were rinsed in water, permeabilized in $0.5 \%$ Triton X-100/PBS, blocked in $1 \%$ BSA/PBS for $30 \mathrm{~min}$ at room temperature, and incubated with BD Living colors AV Monoclonal antibody JL-8 (BD Biosciences) at 1:200 dilution and anti-CD34 biotin-conjugated antibody (RAM34, $\mathrm{BD}$ Pharmingen) at 1:100 dilution for $2 \mathrm{~h}$ at room temperature. After three washes with Tween20-PBS, slides were blocked in $1 \% \mathrm{BSA} / \mathrm{PBS}$ for $30 \mathrm{~min}$ at room temperature and incubated with goat antibody to mouse conjugated with Cy3 (Jackson ImmunoResearch Laboratories) at 1:200 dilution and Streptoavidin FITC-cojugated (BD Pharmingen) at 1:200 dilution for $1 \mathrm{~h}$ at room temperature. After three washes with Tween20/PBS, slides were mounted in DAPI Vectashied medium (Vector Laboratories) and placed at $4^{\circ} \mathrm{C}$. Fluorescence images were obtained in a Leica DM6000B fluorescence microscope with a Cohu CCD camera, and specific filters were used to avoid fluorochrome spectral overlapping: A4 (excitation: BP 360/40, dichromatic mirror 400, suppression filter BP 470/40), Y3 (BP 545/30, 565, BP 610/75), and L5 (BP 480/40, 505, BP 527/30) for DAPI, Cy3, and FITC, respectively. Samples stained with single antibodies and only secondary antibodies were used as negative controls (data not shown).

\section{In vivo imaging of K15-EGFP cells}

Back skins from 2-mo-old K15-EGFP mice were shaved and treated with acetone or TPA (20 nmol in acetone) to induce "mobilization" of epidermal stem cells. At the indicated time points, real-time images at high resolution were obtained through the skin surface from anesthetized animals using the Leica FCM1000 endoscopic confocal microscope (Leica Microsystems) and were visualized with the Leica FCM1000 IC viewer (Leica Microsystems).

\section{Cell sorting and telomere length measurements in K15-EGFP mice}

Eight-week-old K15-EGFP mice were sacrificed, and back skin and tail skin were harvested, fixed overnight in neutral-buffered formalin at $4^{\circ} \mathrm{C}$, dehydrated through graded alcohols and xylene, and embedded in paraffin. Simultaneously, part of the tail skin was collected to obtain keratinocytes (Muñoz et al. 2005). Freshly isolated keratinocyte suspensions were then sorted with a fluorescence-activated cell sorter (FACS) using a MoFlo (DakoCytomation). Cells were excited with a 488-nm laser, and
GFP signals were collected via the FL1 channel (510-550 nm), sorting them in an "enrichment" mode into GFP-positive and GFP-negative cells. The sorted cell suspensions were centrifuged onto microscope slides using a cytospin (Cytospin 3; Thermo Shandon). After air-drying, cells on slides were fixed in methanol/acetic acid (3:1) for $1 \mathrm{~h}$ and dried overnight. Telomere FISH was performed as described calculating the telomere fluorescence of the whole nuclei (Samper et al. 2000; Muñoz et al. 2005). For telomere length quantification on interphase nuclei, Cy3 and DAPI images were captured at $100 \times$ magnification using a COHU CCD camera on a Leica Leitz DMRA microscope, and the telomere fluorescence was integrated and quantified using spot IOD analysis in the TFL-TELO program (Zijlmans et al. 1997) (gift from Dr P. Lansdorp, Vancouver). The telomere fluorescence of individual nuclei was represented by frequency histograms.

In parallel, combined GFP immunostaning/telomere Q-FISH was performed in paraffin-embedded sections of back and tail skin from K15-EGFP mice to quantify telomere length in GFPpositive and GFP-negative cells. Slides were deparaffinized, rehydrated, immersed in $10 \mathrm{mM}$ citrate solution, and epitoperetrieved by three high-power, 5-min microwave pulses. Slides were then rinsed in water, permeabilized in $0.25 \%$ Triton $\mathrm{X}-100 / \mathrm{PBS}$, blocked in $10 \% \mathrm{BSA} / \mathrm{PBS}$ for $30 \mathrm{~min}$ at room temperature, and incubated with BD Living Colors AV Monoclonal Antibody JL-8 (Becton Dickinson) at a 1:250 dilution for $30 \mathrm{~min}$ at room temperature. After three rinses with Tween20/PBS, slides were blocked in $10 \% \mathrm{BSA} / \mathrm{PBS}$ for $30 \mathrm{~min}$ at room temperature and incubated with goat antibody to mouse conjugated with Alexa 488 (1:500; Molecular Probes, Invitrogen) for $30 \mathrm{~min}$ at room temperature. After three rinses with Tween20/PBS, slides were fixed in methanol/acetic acid $(3: 1)$ for $1 \mathrm{~h}$ and dried overnight in the dark. Telomere Q-FISH was performed as described (Gonzalez-Suarez et al. 2000; Muñoz et al. 2005) with minor modifications to preserve GFP immunostaining. DAPI, Cy3, and Alexa488 signals were acquired simultaneously into separate channels using a confocal ultraspectral microscope (Leica TCS-SP5-A-OBS-UV) using a PL APO 20×/0.70 PH2 lens with Leica LAS AF software, and maximum projection from image stacks (10 sections at steps $1.0 \mu \mathrm{m})$ were generated for image quantification.

Flow-FISH telomere length measurements in K15-EGFP mice

Freshly isolated keratinocyte suspensions $\left(\mathrm{EGFP}^{+}\right.$and $\left.\mathrm{EGFP}^{-}\right)$ from K15-EGFP mice were fixed in methanol/acetic acid (3:1), permeabilized with $100 \%$ methanol, and washed in PBS. Cells were blocked in $\mathrm{BSA} / 10 \% \mathrm{PBS}$ for $15 \mathrm{~min}$ at room temperature and incubated with BD Living Colors AV Monoclonal Antibody JL-8 (Becton Dickinson) at 1:250 dilution for $30 \mathrm{~min}$ at room temperature. After two washes in Tween20/PBS, cells were blocked in BSA $/ 10 \%$ PBS for $15 \mathrm{~min}$ at room temperature and incubated with goat antibody to mouse conjugated with Alexa 647 at 1:500 dilution (Molecular Probes, Invitrogen) for $30 \mathrm{~min}$ at room temperature. After two washes in Tween20/PBS, cells were fixed in formaldehyde $0.5 \%$ PBS for $5 \mathrm{~min}$ and washed twice in PBS. Then telomere Flow-FISH was performed as described (Rufer et al. 1998) using a FITC-labeled PNA-tel probe and propidium iodide (Sigma) to counterstain DNA, and was analyzed in a FACScanto cytometer (BD Biosciences). Cells with adequate size and complexity, as determined by forward scatter and side scatter channels, were gated for G0/G1 phase using the propidium iodide signal acquired in the FL2 channel. Their labeling for Alexa 467 was acquired in the FL4 channel and was used to identify GFP-positive and GFP-negative cell populations. The telomere fluorescence as FITC signal was ac- 
quired in FL1 for both cell populations. To compensate for the contribution of cellular autofluorescence, fluorescence values of negative control cells (i.e., cells hybridized in the absence of the FITC PNA-tel probe) were subtracted from every sample. L5178Y-R and L5178Y-S cell lines with known telomere lengths of 10.2 and $79.7 \mathrm{~kb}$ (McIlrath et al. 2001), respectively, were processed in parallel and used to convert fluorescence values into kilobases. Negative controls for each fluorochrome and acquisition settings were established with unstained or singlestained cell populations.

\section{Telomapping of a paraffin-embedded array of human and mouse cell lines}

The cell line array was developed using a range of different cell lines with known telomere length (Canela et al. 2007). Cell lines were cultured, and $4 \times 10^{6}$ cells from each one were used, washed in PBS, fixed in formaldehyde 4\% PBS for 5 min at room temperature, washed twice in PBS, and mixed with melted gelatine (Sigma) /5\% PBS to generate gelatine blocks after polymerization overnight at $4^{\circ} \mathrm{C}$. Gelatine blocks were embedded in paraffin after staining with blue metilene to allow identification within the paraffin block. A small cylindrical cell-containing core of 1-mm diameter was performed in every paraffin block using a Manual Tissue Microarrayer MTA (Beecher) and inserted in a receptor paraffin block separated from each other by

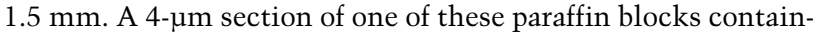
ing every cell line was placed together with skin sections on the same slide, and confocal Q-FISH was performed. Telomapping analysis was carried out on images from skin follicles and interphase nuclei of every cell line, and telomere fluorescence values of the skin follicle compartments were converted into kilobases using these cell lines as a calibration standard with stable and known telomere length (Canela et al. 2007).

\section{TRF-based telomere length analysis of K15-EGFP adult keratinocytes}

Isolated $\mathrm{GFP}^{+}$and $\mathrm{GFP}^{-}$adult epidermal keratinocytes from K15-EGFP mice were sorted as indicated above. A fraction of enriched GFP-negative cells and GFP-positive cells were included in agarose plugs following instructions provided by the manufacturer (Bio-Rad), and TRF analysis was performed as described previously (Blasco et al. 1997).

\section{Quantitative telomere length analyses on K15-EGFP skin sections}

Quantitative image analysis was performed on confocal images using Metamorph (version 6.3r6; Molecular Devices) as described above. The DAPI image was used to define the nuclear area, the Cy3 image was used for telomere fluorescence determinations, and the Alexa488 image was used to identify the GFP-expressing cells. Specific nuclear masks for GFP-positive and GFP-negative cells were generated to allow quantification of telomere fluorescence in the two populations. Images were analyzed as indicated above.

\section{Isolation of newborn keratinocytes}

Two-day-old mice were sacrificed, soaked in Betadine (5 min), in a PBS antibiotics solution (5 $\mathrm{min})$, in $70 \%$ ethanol $(5 \mathrm{~min})$, and in a PBS antibiotics solution (5 min). Newborn keratinocytes were isolated as described (Muñoz et al. 2005).

\section{Isolation of adult keratinocytes}

Two-month-old and 27- to 31-mo-old mice were sacrificed, and tail skin was collected and soaked in Betadine for $5 \mathrm{~min}$, in a PBS antibiotics solution for $5 \mathrm{~min}$, in $70 \%$ ethanol for $5 \mathrm{~min}$, and in PBS antibiotics solution for $5 \mathrm{~min}$. Fresh keratinocytes were obtained as described (Muñoz et al. 2005).

\section{Clonogenic assays}

Mouse keratinocytes $\left(10^{3}\right)$ obtained from 2-d-old mice and $10^{4}$ mouse keratinocytes from 2-mo-old and 27- to 31-mo-old mice were seeded onto mitomycin C-treated $(10 \mu \mathrm{g} / \mathrm{mL}, 2 \mathrm{~h}) \mathrm{J} 2-3 \mathrm{~T} 3$ fibroblasts $\left(10^{5}\right.$ per well, six-well dishes) and grown at $37^{\circ} \mathrm{C} / 5 \%$ $\mathrm{CO}_{2}$ in Cnt-02 medium (CELLnTEC Advanced Cell Systems AG). After $10 \mathrm{~d}$ of cultivation, dishes were rinsed twice with PBS, fixed in $10 \%$ formaldehyde, and then stained with $1 \%$ Rhodamine B to visualize colony formation. Colony size and number were measured using three dishes per experiment.

\section{Statistical analysis}

A Wilcoxon's ram sum test was used to calculate the statistical significance of the observed differences in the different assays. Microsoft Excel 2001 and Graphpad Instat version 3.05 were used for the calculations.

In all cases, differences are significant for $P<0.05$, very significant for $P<0.01$, and highly significant for $P<0.001$.

\section{Acknowledgments}

We thank R. Serrano and E. Santos for mouse care and genotyping, and the Comparative Pathology, Molecular Imaging, and Confocal Microscopy Units at the CNIO for help. Thanks to O. Fernandez-Capetillo, E. Moreno, M. Serrano, and members of M.A.B.'s laboratory for critical reading of the manuscript. I.F. is a "Ramon y Cajal" senior scientist. A.C. and E.V. are supported by the European Union. M.A.B.'s laboratory is funded by the MCyT (SAF2005-00277, GEN2001-4856-C13-08), by the Regional Government of Madrid (GR/SAL/0597/2004), European Union (TELOSENS FIGH-CT-2002-00217, INTACT LSHC-CT2003-506803, ZINCAGE FOOD-CT-2003-506850, RISC-RAD FI6R-CT-2003-508842, MOL CANCER MED LSHC-CT-2004502943), and the Josef Steiner Cancer Research Award 2003.

\section{References}

Alvarez-Buylla, A. and Lim, D.A. 2004. For the long run: Maintaining germinal niches in the adult brain. Neuron 41: 683686.

Barker, N., van Es, J.H., Kuipers, J., Kujala, P., van den Born, M., Cozijnsen, M., Haegebarth, A., Korving, J., Begthel, H., Peters, P.J., et al. 2007. Identification of stem cells in small intestine and colon by marker gene Lgr5. Nature 449: 10031007.

Barrandon, Y. and Green, H. 1987. Three clonal types of keratinocyte with different capacities for multiplication. Proc. Natl. Acad. Sci. 84: 2302-2306.

Blasco, M.A. 2005. Telomeres and human disease: Cancer, ageing and beyond. Nat. Rev. Genet. 6: 611-622.

Blasco, M.A., Lee, H.-W., Hande, P., Samper, E., Lansdorp, P., DePinho, R., and Greider, C.W. 1997. Telomere shortening and tumor formation by mouse cells lacking telomerase RNA. Cell 91: 25-34.

Braun, K.M., Niemann, C., Jensen, U.B., Sundberg, J.P., Silva- 
Vargas, V., and Watt, F.M. 2003. Manipulation of stem cell proliferation and lineage commitment: Visualisation of label-retaining cells in wholemounts of mouse epidermis. Development 130: 5241-5255.

Brinster, R.L. 2002. Germline stem cell transplantation and transgenesis. Science 296: 2174-2176.

Canela, A., Vera, E., Klatt, P., and Blasco, M.A. 2007. Highthroughput telomere length quantification by FISH and its application to human population studies. Proc. Natl. Acad. Sci. 104: 5300-5305.

Chan, S.W. and Blackburn, E.H. 2002. New ways not to make ends meet: Telomerase, DNA damage proteins and heterochromatin. Oncogene 21: 553-563.

Collins, K. and Mitchell, J.R. 2002. Telomerase in the human organism. Oncogene 21: 564-579.

Cotsarelis, G., Sun, T.T., and Lavker, R.M. 1990. Label-retaining cells reside in the bulge area of pilosebaceous unit: Implications for follicular stem cells, hair cycle, and skin carcinogenesis. Cell 61: 1329-1337.

Coviello-McLaughlin, G.M. and Prowse, K.R. 1997. Telomere length regulation during postnatal development and ageing in Mus spretus. Nucleic Acids Res. 25: 3051-3058.

de Lange, T. 2005. Shelterin: The protein complex that shapes and safeguards human telomeres. Genes \& Dev. 19: 21002110.

Flores, I., Cayuela, M.L., and Blasco, M.A. 2005. Effects of telomerase and telomere length on epidermal stem cell behavior. Science 309: 1253-1256.

Flores, I., Benetti, R., and Blasco, M.A. 2006. Telomerase regulation and stem cell behaviour. Curr. Opin. Cell Biol. 18: 254-260.

Fuchs, E., Tumbar, T., and Guasch, G. 2004. Socializing with the neighbours: Stem cells and their niche. Cell 116: 769778.

Gage, F.H. 2000. Mammalian neural stem cells. Science 287: $1433-1438$.

García-Cao, I., García-Cao, M., Tomás-Loba, A., Martín-Caballero, J., Flores, J.M., Klatt, P., Blasco, M.A., and Serrano, M. 2006. Increased p53 activity does not accelerate telomeredriven aging. EMBO Rep. 7: 546-552.

Gonzalez-Suarez, E., Samper, E., Flores, J.M., and Blasco, M.A. 2000. Telomerase-deficient mice with short telomeres are resistant to skin tumorigenesis. Nat. Genet. 26: 114-117.

Gregorieff, A., Pinto, D., Begthel, H., Destree, O., Kielman, M., and Clevers, H. 2005. Expression pattern of Wnt signaling components in the adult intestine. Gastroenterology 129: 626-638.

Guan, K., Nayernia, K., Maier, L.S., Wagner, S., Dressel, R., Lee, J.H., Nolte, J., Wolf, F., Li, M., Engel, W., et al. 2006. Pluripotency of spermatogonial stem cells from adult mouse testis. Nature 440: 1199-1203.

Harrington, L. 2004. Does the reservoir for self-renewal stem from the ends? Oncogene 23: 7283-7289.

Ito, M., Liu, Y., Yang, Z., Nguyen, J., Liang, F., Morris, R.J., and Cotsarelis, G. 2005. Stem cells in the hair follicle bulge contribute to wound repair but not to homeostasis of the epidermis. Nat. Med. 11: 1351-1354.

Lansdorp, P.M. 2005. Role of telomerase in hematopoietic stem cells. Ann. N. Y. Acad. Sci. 1044: 220-227.

Lavker, R.M., Tseng, S.C., and Sun, T.T. 2004. Corneal epithelial stem cells at the limbus: Looking at some old problems from a new angle. Exp. Eye Res. 78: 433-446.

Lehrer, M.S., Sun, T.T., and Lavker, R.M. 1998. Strategies of epithelial repair: Modulation of stem cell and transit amplifying cell proliferation. J. Cell Sci. 111: 2867-2875.

Leri, A., Malhotra, A., Liew, C.C., Kajstura, J., and Anversa, P.
2000. Telomerase activity in rat cardiac myocytes is age and gender dependent. J. Mol. Cell. Cardiol. 32: 385-390.

Marshman, E., Booth, C., and Potten, C.S. 2002. The intestinal epithelial stem cell. Bioessays 24: 91-98.

Mason, P.J., Wilson, D.B., and Bessler, M. 2005. Dyskeratosis congenita-A disease of dysfunctional telomere maintenance. Curr. Mol. Med. 5: 159-170.

McIlrath, J., Bouffler, S.D., Samper, E., Cuthbert, A., Wojcik, A., Szumiel, I., Bryant, P.E., Riches, A.C., Thompson, A., Blasco, M.A., et al. 2001. Telomere length abnormalities in mammalian radiosensitive cells. Cancer Res. 61: 912-915.

Meeker, A.K. and De Marzo, A.M. 2004. Recent advances in telomere biology: Implications for human cancer. Curr. Opin. Oncol. 16: 32-38.

Meeker, A.K., Gage, W.R., Hicks, J.L., Simon, I., Coffman, J.R., Platz, E.A., March, G.E., and De Marzo, A.M. 2002. Telomere length assessment in human archival tissues: Combined telomere fluorescence in situ hybridization and immunostaining. Am. J. Pathol. 160: 1259-1268.

Meeker, A.K., Hicks, J.L., Gabrielson, E., Strauss, W.M., De Marzo, A.M., and Argani, P. 2004. Telomere shortening occurs in subsets of normal breast epithelium as well as in situ and invasive carcinoma. Am. J. Pathol. 164: 925-935.

Moore, K.A. and Lemischka, I.R. 2006. Stem cells and their niches. Science 311: 1880-1885.

Morris, R.J., Liu, Y., Marles, L., Yang, Z., Trempus, C., Li, S., Lin, J.S., Sawicki, J.A., and Cotsarelis, G. 2004. Capturing and profiling adult hair follicle stem cells. Nat. Biotechnol. 22: 411-417.

Muñoz, P., Blanco, R., Flores, J.M., and Blasco, M.A. 2005. XPF nuclease-dependent telomere loss and increased DNA damage in mice overexpressing TRF2 result in premature aging and cancer. Nat. Genet. 10: 1063-1071.

Oshima, H., Rochat, A., Kedzia, C., Kobayashi, K., and Barrandon, Y. 2001. Morphogenesis and renewal of hair follicles from adult multipotent item cells. Cell 104: 233-245.

Prowse, K.R. and Greider, C.W. 1995. Developmental and tissue-specific regulation of mouse telomerase and telomere length. Proc. Natl. Acad. Sci. 92: 4818-4822.

Ramirez, R.D., Wright, W.E., Shay, J.W., and Taylor, R.S. 1997. Telomerase activity concentrates in the mitotically active segments of human hair follicles. J. Invest. Dermatol. 108: 113-117.

Rufer, N., Dragowska, W., Thornbury, G., Roosnek, E., and Lansdorp, P.M. 1998. Telomere length dynamics in human lymphocyte subpopulations measured by flow cytometry. Nat. Biotechnol. 16: 743-747.

Samper, E., Goytisolo, F.A., Slijepcevic, P., van Buul, P.P., and Blasco, M.A. 2000. Mammalian Ku86 protein prevents telomeric fusions independently of the length of TTAGGG repeats and the G-strand overhang. EMBO Rep. 1: 244-252.

Sarin, K.Y., Cheung, P., Gilison, D., Lee, E., Tennen, R.I., Wang, E., Artandi, M.K., Oro, A.E., and Artandi, S.E. 2005. Conditional telomerase induction causes proliferation of hair follicle stem cells. Nature 436: 1048-1052.

Tumbar, T., Guasch, G., Greco, V., Blanpain, C., Lowry, W.E., Rendl, M., and Fuchs, E. 2004. Defining the epithelial stem cell niche in skin. Science 303: 359-363.

Zijlmans, J.M., Martens, U.M., Poon, S.S., Raap, A.K., Tanke, H.J., Ward, R.K., and Lansdorp, P.M. 1997. Telomeres in the mouse have large inter-chromosomal variations in the number of T2AG3 repeats. Proc. Nat1. Acad. Sci. 94: 7423-7428. 


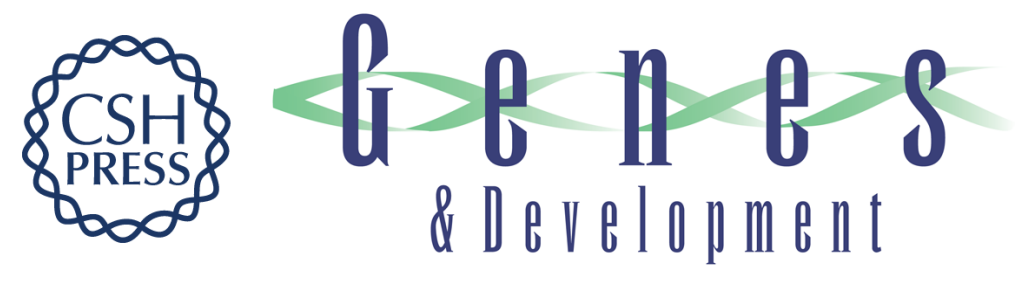

\section{The longest telomeres: a general signature of adult stem cell compartments}

Ignacio Flores, Andres Canela, Elsa Vera, et al.

Genes Dev. 2008, 22: originally published online February 18, 2008

Access the most recent version at doi:10.1101/gad.451008

\section{Supplemental http://genesdev.cshlp.org/content/suppl/2008/02/19/gad.451008.DC1 \\ Material}

References This article cites 44 articles, 14 of which can be accessed free at:

http://genesdev.cshlp.org/content/22/5/654.full.html\#ref-list-1

\section{License}

Email Alerting

Receive free email alerts when new articles cite this article - sign up in the box at the top

Service

right corner of the article or click here.

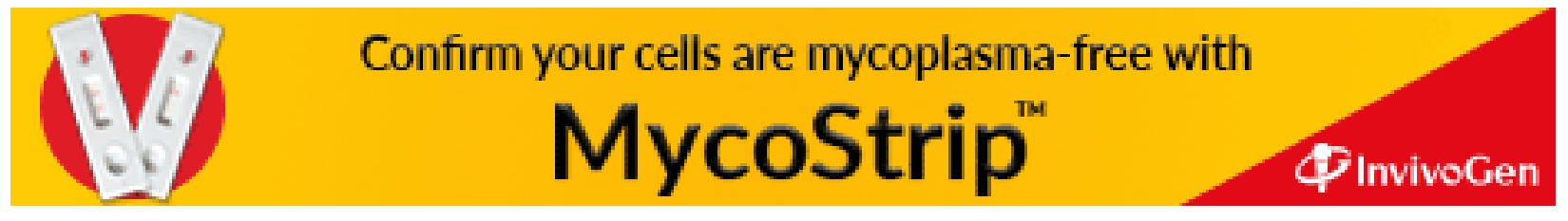

